\title{
Multiple Material Property Characterization Using Induced Currents and Atomic Force Microscopy
}

\author{
Vijay Nalladega ${ }^{1}$, Shamachary Sathish ${ }^{1}$, \\ Kumar V. Jata ${ }^{2}$ and Mark P. Blodgett ${ }^{2}$ \\ ${ }^{1}$ Structural Integrity Division, University of Dayton Research Institute, Dayton, OH \\ ${ }^{2}$ Air Force Research Laboratory, Wright-Patterson Air Force Base, Dayton
}

USA

\section{Introduction}

The invention of atomic force microscope (AFM) by Binnig and his co-workers (Binnig et al., 1986) has led to the imaging of conducting and insulating surfaces with nanometer scale resolution. The AFM measures very small forces (less than $\mathrm{nN}$ ) between a cantilever-tip and the sample surface. When the tip is brought near the surface, the interaction forces between the tip and the sample cause the cantilever to deflect.A topographic image of the surface is obtained by raster scanning the tip across the sample surface and using the interaction force as a parameter for a feedback electronics system which maintains the force at a constant set value. Since the invention of the AFM, it has become a popular tool for surface characterization and is now routinely used in many industries and academic research labs with applications in several research areas.

The initial applications of the AFM were focused on high resolution surface topography imaging of materials. Though it provides high resolution topography images, it cannot provide physical property information. This has led to the development of AFM methods designed to image simultaneously physical properties with topography.Tapping mode AFM, magnetic force microscopy (MFM) (Hartmann, 1999), electric force microscopy (Bluhm et al., 1997; Nyffenegger et al., 1997) are some of the examples. Further imaging modes were developed later to image elastic stiffness (Burnham et al., 1995; Dinelli et al., 1999; Nalladega et al., 2008; Rabe \& Arnold, 1994; Yamanaka et al., 1994), surface potential (Nonnenmacher et al., 1991), thermal conductivity (Gu et al., 2002), dielectric properties (Stern et al., 1988), and optical properties (Betzig et al., 1991). The families of instruments based on AFM are known as scanning probe microscopes (SPM). All SPM techniques are based on the same principle, i.e., scanning a probe in the near-field across the sample surface. The techniques of SPM differ only in the selective detection of different sample-probe interactions among the many kinds of interactions between the probe and the sample. For example, if an electrical potential difference is externally applied, electrostatic interactions can be imaged by utilizing a conductive probe. Similarly, a magnetic probe is used to image magnetostatic interactions between the magnetic probe and ferromagnetic surface. The unique combination of nanoscale 
resolution and broad applicability has led to the proliferation of SPM techniques into virtually all areas of nanometer-scale science and technology.

To measure electrical properties using AFM, a bias voltage is applied between a conducting probe and the sample and the resulting electrical interactions (electrostatic forces, electric currents, resistance, and capacitance etc.) are measured. Depending on the type of the electrical interaction, different electrical property can be measured. Various techniques have been developed based on measuring these interactions to image electrical properties. Electrostatic force microscopy (Bluhm et al., 1997; Nyffenegger et al., 1997), conducting AFM (Oh \& Nemanich, 2002; Olbrich et al., 1998), tunneling AFM (Gautier et al, 2004; Ruskell et al., 1996), scanning capacitance microscopy (Matey \& Blanc, 1985; Williams, 1999), surface potential imaging (Weaver \& Abraham, 1991), and piezoresponse force microscopy (Franke et al., 1994; Gruverman et al., 1996) are some of the widely used AFM techniques for studying electrical properties. To obtain an image of the electrical property, the probe measures the interactions at each location by moving from one discrete location to the next across the scan area. Therefore, these techniques are quite time consuming. Moreover, a bias voltage is always applied between the sample and the tip requiring a conducting tip to perform the measurements. In addition, some of these techniques require a physical contact between the tip and sample.

Electrical properties can also be measured using electromagnetic induction. When a conductor is placed in a time varying magnetic field, currents are induced in the conductor by the magnetic field. These currents are known as eddy currents. Since currents are induced in the conductor, no physical contact between the source and the conductor is needed. Several techniques have been developed based on eddy currents to develop electrodeless methods to measure electrical properties of materials. In these methods, the sample is placed in the field of a coil excited using an AC source. The time-varying magnetic field induces currents in the sample. The induced currents produce a magnetic field opposing the primary field, which changes the impedance of the coil. The electrical impedance can be used to determine the resistivity of the sample.

In addition to the measurement of electrical conductivity, eddy currents are also used in nondestructive evaluation (NDE) of defects in materials. It is well known that defects in a material modify the flow of induced currents in the vicinity of the defect. Consequently, the electrical conductivity around the defect is also different. This fact has been effectively utilized for NDE applications (Libby, 1971). In a typical eddy current testing method, a coil is located as near as possible to the sample being tested and is excited with a time-varying magnetic field at a given frequency. When the coil is scanned across a defect, the impedance of the coil is modified. Therefore, by monitoring the changes in impedance of the coil, it is possible to detect defects in the material. This methodology has been used for NDE applications as well as for the measurement of electrical and magnetic properties under various environmental conditions. It is possible to generate electrical conductivity images by scanning the coil in a raster pattern (Kirby \& Lareau, 1997). The spatial resolution in eddy current imaging is dependent on the diameter of the coil and the best spatial resolution is about $50 \mu \mathrm{m}$ (Karpen et al., 1999). Eddy current methods are sensitive to small changes in electrical and magnetic properties. Thus, small changes in the properties can be detected. However, eddy current methods are essentially comparison methods and it is not possible to get absolute values of electrical conductivity. The electrical conductivity is always given in terms of conductivity of a calibrated standard. 
The invention of AFM has enabled the development of eddy current microscopy techniques with better spatial resolution than that of conventional eddy current imaging systems. In magnetic force microscopy (MFM), a magnetic probe is oscillated above a magnetic surface. The oscillating magnetic probe generates eddy currents. This concept was used in the development of an MFM based eddy current microscopy (Hoffmann et al., 1998). This technique was used to image local variations in electrical conductivity of a sample consisting of $\mathrm{TiC}$ precipitates in $\mathrm{Al}_{2} \mathrm{O}_{3}$ matrix with nanometer scale resolution. However, since the magnetic field of an MFM tip is small, this technique is not suitable to image small variations in conductivity. The sensitivity of this technique was improved by using large magnetic fields from a tip made ofpermanent magnet (Lantz et al., 2001). This resulted in increased sensitivity but reduced the spatial resolution down to hundreds of nanometers.

From the above discussion it is evident that it is difficult to achieve both high resolution and high sensitivity to local variations in electrical conductivity using eddy current microscopy by MFM. To improve the sensitivity, a flexible cantilever capable of detecting small variations in the forces can be employed. However, in MFM techniques, a stiffer cantilever, vibrated at its resonant frequency, is used in order to make the cantilever sensitive only to the long-range magnetic forces. But by using a stiffer cantilever, it is difficult to measure small forces generated due to very small variations in the electrical conductivity. The magnetic tips used in MFM have small magnetic field strength. Therefore, the eddy current density that can be induced in the sample material is limited.

The above considerations led to the development of a new high-resolution, non-contact electrical conductivity imaging technique. The technique, called scanning eddy current force microscopy (SECFM), combines the principles of eddy currents and AFM to achieve high spatial resolution and high sensitivity to variations in electrical conductivity on nanoscale. The technique is based on a simple principle- detecting the magnetic forces due to the interactions between a magnetic probe and the magnetic field generated by eddy currents in a conducting sample. To achieve higher sensitivity, a small electromagnetic coil is excited near the sample and eddy currents are generated in the sample. Further sensitivity is achieved by employing soft cantilevers $(0.1 \mathrm{~N} / \mathrm{m})$ to detect small changes in electrical conductivity. The magnetic field due to eddy currents interacts with the static magnetic field of the probe resulting in magnetic forces. The magnitude of the magnetic forces generated is directly proportional to the electrical conductivity of the sample. The deflection of the cantilever due to the forces is measured and analyzed by a custom-built electronic instrumentation to generate surface topography and electrical conductivity images simultaneously. Since currents are induced, bias voltage is not required between the probe and the sample thus removing the need of conducting tips. The electrical conductivity images are obtained in non-contact fashion. The new instrument has a spatial resolution of $20-25 \mathrm{~nm}$. The instrument is used to characterize electrical properties of different materials. The contrast mechanisms in different materials are explained based on the variation of the magnetic forces caused by eddy currents in different materials. In addition to the electrical properties, we also show that by doing small modifications to the system, it is possible to characterize magnetic, magneto-elastic properties. The advantages, limitations and possible applications of the instrument in materials characterization and nano NDE are discussed. 


\section{Theory and development of SECFM}

The central element of an AFM is the force sensor. For maximum sensitivity to electrical conductivity variations, the sensor should detect small forces generated by the eddy currents. Therefore, it is necessary to select a cantilever spring constant capable of measuring small forces. In order to do so, however, magnetic forces between the probe and eddy currents should be known first. Therefore, a theoretical model is first used to calculate the eddy current forces in a typical metal. The model is used to describe electrodynamic interactions between eddy currents and the probe. Based on the calculated forces, a suitable cantilever is selected.

\subsection{Theory}

Eddy current fields are considered to be quasi-static fields. Quasi-static condition requires that the wavelength $\lambda$ of the field is much greater than the dimensions of the conductor (Landau \& Lifshitz, 1960). The magnetic field $\mathbf{H}$ generated by the eddy currents in a nonmagnetic conductor is

$$
\nabla^{2} \vec{H}=\sigma \mu \frac{\partial \vec{H}}{\partial t}
$$

where $\sigma$ is the electrical conductivity, $\mu=\mu_{0} \mu_{\mathrm{r}}, \mu_{0}$ is the magnetic permeability of the free space, and $\mu_{\mathrm{r}}$ is the relative permeability. In a variable field of frequency $\omega$, all quantities depend on the time through a factor ej ${ }^{\mathrm{\omega t}}$. The magnetic field intensity, therefore can be written as

$$
H(t)=H^{*} e^{j \omega t}
$$

Substituting Eq. (2) into Eq. (1)

$$
\nabla^{2} H=j \omega \sigma \mu \mathrm{H}
$$

or

$$
=k^{2} \mathrm{H}
$$

where $k^{2}=j \omega \sigma \mu$ and $j^{2}=-1$. The constant $k$ is connected with the penetration depth of an electromagnetic wave. The eddy currents tend to flow near the surface of the conductor. The eddy current density in a conductor is strongest near the source of the field and exponentially decreases with increasing thickness of the conductor. This effect is known as skin effect and is dependent on many factors such as electrical conductivity, frequency of the source, and magnetic properties (Libby, 1971).

\subsubsection{Magnetic field due to eddy currents in a conductor}

Figure 1 shows the schematic of a non-magnetic electrically conducting sample placed in the field of an electromagnetic field with a magnetic probe above its surface. The electromagnetic field is excited by a small cylindrical coil of diameter $a$, with $\mathrm{N}$ number of turns. The diameter of the coil is smaller compared to the lateral dimensions of the sample. The thickness of the sample, $t$, is very small compared to the diameter of the coil. The timevarying AC signal through the coil produces a uniform magnetic field within the diameter 
of the coil. The normal component of the magnetic field is designated as $\mathbf{B}_{0}$ as shown in Fig. 1. The oscillating normal component of the magnetic field produces eddy currents in the conductor. The magnetic field generated by the eddy currents in the sample is assumed to be uniform within the diameter of the coil.

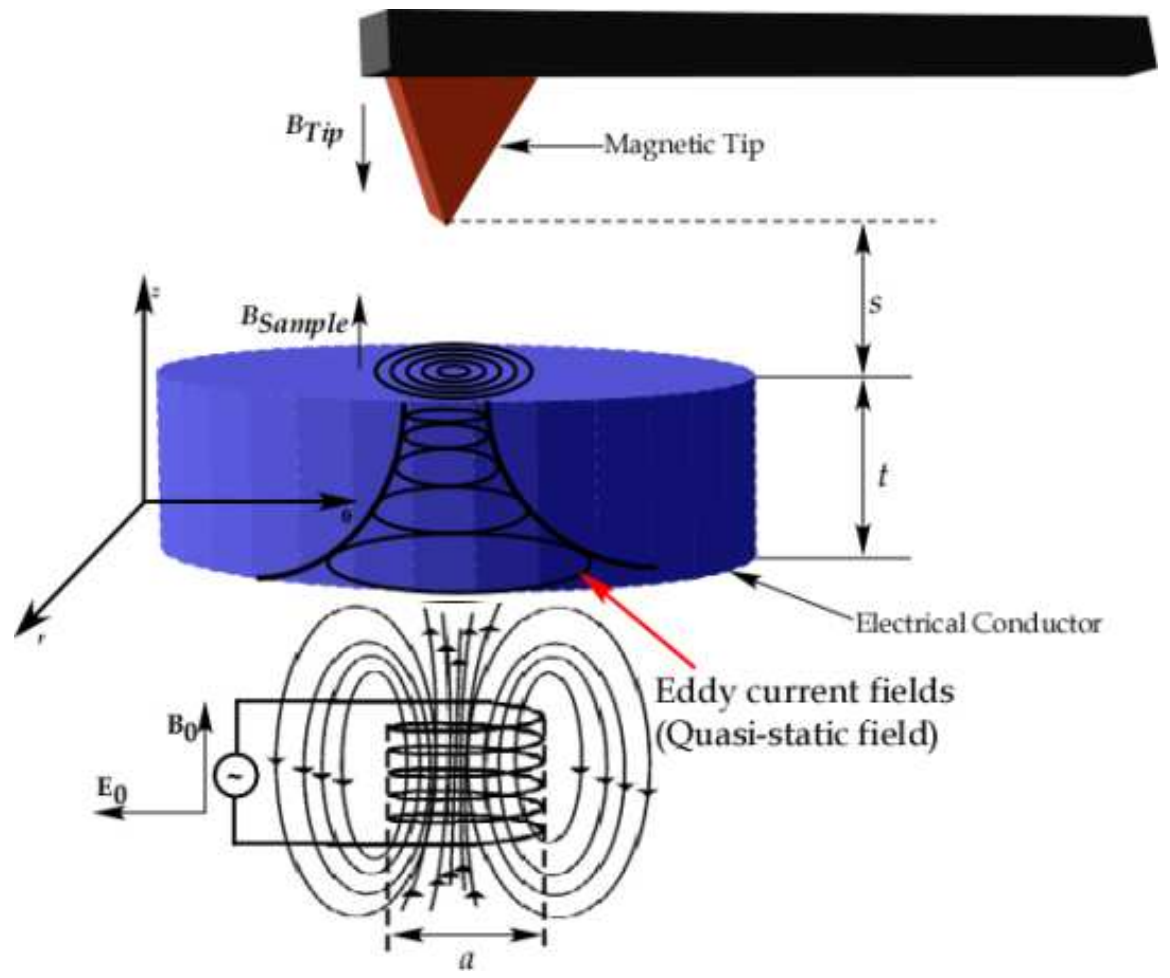

Fig. 1. Schematic of a non-magnetic electrical conductor placed in an oscillating electromagnetic field. A magnetic tip attached to a cantilever is positioned above the sample.

The relationship between eddy current densityJ and magnetic field $\mathbf{H}$ is given by

$$
\begin{gathered}
\nabla \times H=J \\
\nabla \times J=-\left(j \omega \mu_{0} \sigma H_{z}+j \omega \sigma B_{0}\right) e_{z}
\end{gathered}
$$

where $\mathrm{H}_{\mathrm{z}}$ is the normal component of the magnetic field due to eddy currents and $\mathrm{e}_{\mathrm{z}}$ a unit vector in the z-direction. The electrical conductivity is assumed to be constant along the thickness of the sample. In cylindrical coordinates, the eddy current density $\mathbf{J}$ can be represented by a scalar potential $u(r)$ (Poltz, 1983) as

$$
J=\frac{1}{t}(\nabla \times u(r)) e_{z}
$$

Substituting Eq. (7) in Eq.(5), 


$$
\nabla \times\left(H-\frac{1}{t} u(r)\right) e_{z}=0
$$

The normal component of the magnetic field due to eddy currents $\mathrm{H}_{\mathrm{z}}$ is written as

$$
H_{z}=\frac{1}{t} u(r)
$$

In the experiments, we used a magnetic tip with a diameter $d$, positioned above the sample to measure the magnetic interactions generated by the eddy currents. Therefore, the magnetic interactions occur over a region equal to the diameter of the tip. Therefore, the scalar potential $u(r)$ should be evaluated over the region equivalent to the tip diameter. Substituting Eq. (7) in Eq. (6), the scalar potential function evaluated in $0 \leq r<a$ is written as

$$
\frac{\partial^{2} u}{\partial r^{2}}+\frac{1}{r} \frac{\partial u}{\partial r}=j \omega \mu_{0} \sigma u(r)+j \omega \sigma t B_{0}
$$

The solution (Krakowski, 1982) to the above equation is

$$
u(r)=\frac{t B_{0}}{\mu_{0}}\left(\frac{I_{0}(k r)}{I_{0}(k a)}-1\right), 0 \leq r \leq a
$$

where $k=\sqrt{\omega \mu_{0} \sigma} e^{\left(j \frac{\pi}{4}\right)}, \mathrm{I}_{0}(k r)$ and $\mathrm{I}_{0}(k a)$ are zeroth order Bessel function. The constant $k$ is related to the penetration depth of the electromagnetic waves into the sample and is an important factor considered in eddy current testing. The constant $k$ can be written in terms of penetration depth, $\delta$ as

$$
\begin{gathered}
k=\frac{1+j}{\delta} \\
\delta=\sqrt{\frac{2}{\omega \sigma \mu_{0}}}
\end{gathered}
$$

Using the scalar potential function, the normal component of the secondary magnetic field $\mathrm{H}_{\mathrm{z}}$ can be calculated using Eq. (9). This magnetic field interacts with the static magnetic field of the tip.

\subsubsection{Magnetic field of the tip}

A pyramidal shaped magnetic coated tip attached is used as a force sensor in our experiments. Let $\mathbf{M}$ be the magnetization of the magnetic tip. Let the magnetic field generated by a magnetization Mof the tip (Hirsekorn et al., 1999) is given by $\mathrm{H}_{\text {tip }}$. Then,

$$
H_{\text {tip }}(s)=\frac{1}{4 \pi} \oiiint d^{3} r_{i}\left(\frac{3 M\left(s-r_{i}\right.}{\left|s-r_{i}\right|^{5}}\left(s-r_{i}-\frac{M}{\left|s-r_{i}\right|^{3}}\right)\right.
$$


where $r_{i}$ is the location within the magnetic coating of volume $\mathrm{V}, d$ is the thickness of the magnetic coating of the tip, and $s$ is distance between the tip and sample surface. The tip can be modeled using either monopole or dipole approximation (Hirsekorn et al., 1999). Since the dimensions of the tip are large compared to the distance between the tip and sample, a monopole approximation is used. In this case, $\mathrm{H}_{\text {tip }}$ can be written as (Hirsekorn et al., 1999)

$$
H_{t i p M}=-\frac{q}{4 \pi} \frac{r_{i}}{s^{3}}
$$

where $\mathrm{q}$ is the monopole moment of the tip magnetized along the $\mathrm{z}$-axis and given by

$$
q=\frac{M V}{l}
$$

where $l$ is the length of the tip.

The eddy current forces can be determined once the magnetic field strengths of both secondary magnetic field due to eddy currents and the magnetic tip are known. The eddy current force as defined in this work is the difference in the magnetic force measured by the tip before and after the introduction of the sample. When there is no sample between the coil and the tip, the interaction is between the magnetic fields of the coil $\left(\mathbf{B}_{0}\right)$ and the tip $\left(\mathbf{B}_{\text {Tip }}\right)$. When a conductor is introduced between the tip and coil, the eddy currents screen the magnetic field and decrease the force on the tip. The difference between the two forces is the eddy current force.

The eddy current force for a typical metal $\left(\sigma=10^{7}(\Omega \mathrm{m})^{-1}\right)$ is calculated based on the theoretical model.The frequency of excitation is taken to be $100 \mathrm{kHz}$. The coil is taken with 100 turns of copper wire with $6 \mathrm{~mm}$ diameter. The magnetic field, $\mathrm{B}_{0}$ when a current of 86 $\mathrm{mA}$ flows through is approximately $17 \mathrm{kA} / \mathrm{m}$. The thickness and volume of the magnetic coating are taken as $60 \mathrm{~nm}$ and $4.2 \times 10^{-19} \mathrm{~m}^{3}$ respectively. The magnetization of the coating, $\mathbf{M}$ is $114 \mathrm{kA} / \mathrm{m}$ (Wadas \& Hug, 1992). The theoretical eddy current forceis calculated to be 50 $\mathrm{pN}$ at a separation of $100 \mathrm{~nm}$ between probe and tip.

\subsection{Scanning eddy current force microscope}

Figure 2 shows a schematic diagram of the experimental setup used for electrical conductivity imaging. A Digital Instruments Dimension 3000 was modified for the purpose of electrical conductivity imaging (Nalladega et al., 2008b). The maximum scan area of the scanner in this system is $100 \mu \mathrm{m}$. Magnetic tips used in MFM have a spring constant of greater than $2 \mathrm{~N} / \mathrm{m}$. Based on the theoretical calculation, the spring constant of a cantilevers should be less than $0.5 \mathrm{~N} / \mathrm{m}$. A magnetic tip-cantilever with spring constant of $0.1 \mathrm{~N} / \mathrm{m}$ (Veeco Probes, Model MSNC-MFM) was used as the probe The cantilever is a V-shaped cantilever made of $\mathrm{Si}_{3} \mathrm{~N}_{4}$ with a resonant frequency of $25 \mathrm{kHz}$ with a length of $153 \mu \mathrm{m}$ and width of $44 \mu \mathrm{m}$. The tip is coated with a thin layer (thickness $\sim 10-250 \mathrm{~nm}$ ) of Co/Cr and a radius of $10 \mathrm{~nm}$. The force sensitivity of the cantilever is well within the range of calculated theoretical eddy current forces.

For the purpose of generating eddy currents in the sample, an air-core electromagnetic coil is designed with a radius of $6 \mathrm{~mm}$ and 100 turns of 36 gauge copper wire. The sample is 
placed on the coil and one face of the sample faces the circular end of the coil. The opposite face of the sample faces the cantilever with the magnetic film coated tip. The coil is excited by a sinusoidal radio frequency signal from a signal generator (HP 33120A) with appropriate frequency and amplitude. The strength of the eddy currents exponentially decreases as the distance increases from the coil into the sample. The circular eddy currents in the sample produce a magnetic field that is opposing the magnetic field of the coil. The combined electromagnetic force of oscillating magnetic field and the eddy currents in the conducting sample produces oscillations of the magnetic tip-cantilever. For the purpose of measuring eddy current forces, the cantilever-tip is positioned over the sample. The oscillation amplitude of the cantilever due to eddy current forces is detected by the fourquadrant photo-detector. The eddy current force is then determined by multiplying the amplitude with the spring constant of the cantilever. The amplitude of the oscillation of the cantilever is proportional to the conductivity of the sample material.

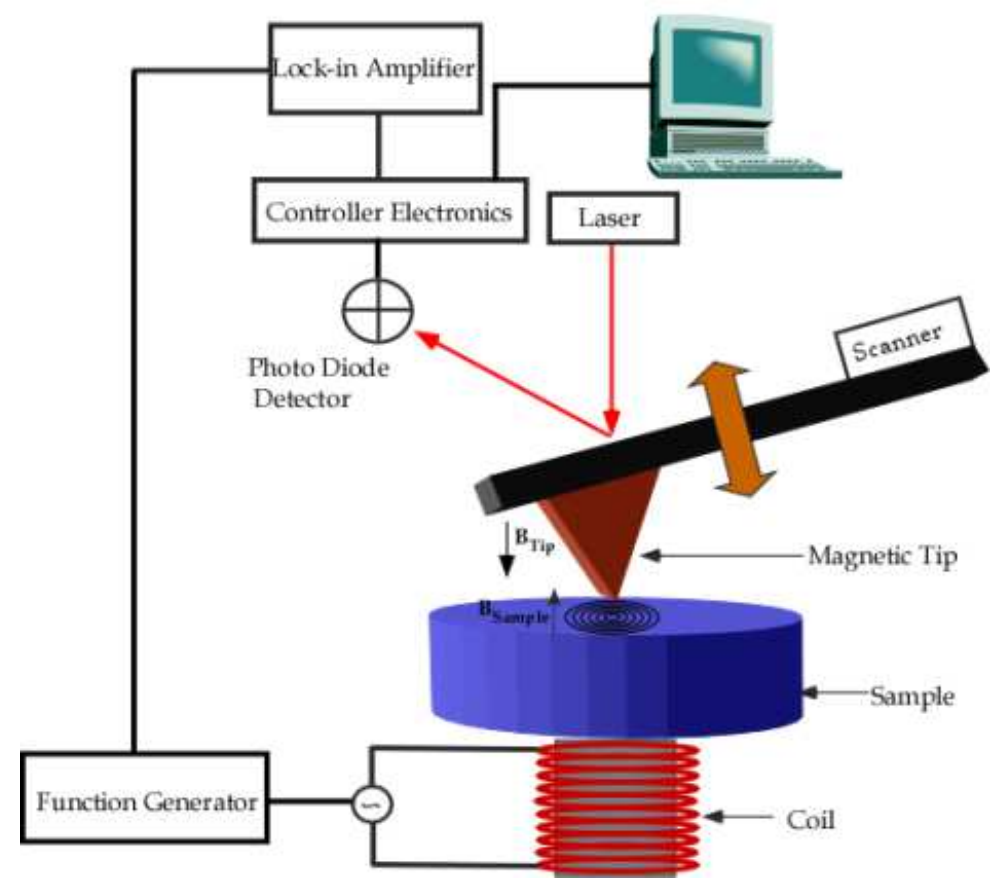

Fig. 2. A schematic diagram of the scanning eddy current force microscopy system

The electrical conductivity images were obtained using lift mode of the AFM. Lift mode allows the imaging of relatively weak but long-range interactions while minimizing the effects of topography. Measurements are taken in two passes across each scan line. In the first pass,the surface topography is obtained on one trace and retrace.The tip is then raised to the lift scan height and a second trace and retrace is obtained while maintaining constant separation between the tip and the surface topography. In the second pass, long range tipsample interactions are measured. In the case of electrical conductivity imaging, the interactions are long-range magnetic forces between the magnetic tip and eddy currents in 
the sample. Therefore, electrical conductivity imaging is performed in non-contact fashion. The output of the photo-detector and the input signal to the coil are fed into a lock-in amplifier (SR 844). The lock-in amplifier measures the differential amplitude and the difference in the phase between the signal to the coil and the photo-detector signal. The difference in amplitude and the phase detected by the lock-in amplifier is proportional to the electrical conductivity of the sample under the magnetic tip. The output of the lock-in amplifier and the controller electronics of the AFM are used to generate surface topography and electrical conductivity images sample simultaneously.

\section{Characterization of electrical properties}

\subsection{Experimental measurement of eddy current forces}

Single crystal metallic samples of copper, cadmium, aluminum and polycrystalline platinum were chosen for the purpose of measuring eddy current forces. The electrical conductivity of these samples are respectively $5.961 \times 10^{7}(\Omega \mathrm{m})^{-1}, 3.745 \times 10^{7}(\Omega \mathrm{m})^{-1}, 1.36 \times 10^{7}(\Omega \mathrm{m})^{-1}$, and 0.94 $\mathrm{x} 10^{7}(\Omega \mathrm{m})^{-1}$. The eddy current force on each of the samples was measured in the following way. In the first step, an insulator was placed in the field of the coil excited with AC signal. The force on magnetic tip-cantilever due to coil's magnetic field was measured ( $\left.F_{\text {Ins }}\right)$. In the second step, the insulator was replaced by the metallic sample and the force is measured $\left(\mathrm{F}_{\mathrm{M}}\right)$. The difference between the two forces $\left[\mathrm{F}_{\text {Ins }}-\mathrm{F}_{\mathrm{M}}\right]$ is the eddy current force in the metallic sample and is directly dependent on the electrical conductivity of the metal. To determine the eddy current forces, the magnetic tip was positioned at a distance of $50 \mathrm{~nm}$ from the surface of the sample. The frequency of the excitation was chosen to be the resonant frequency of the cantilever while positioned over the sample.

Figure 3 compares the oscillation amplitudes of the AFM cantilever while positioned over different metallic samples. The frequency of the excitation was $82 \mathrm{kHz}$. It can be seen that the peak to peak amplitude is different for different metals. Platinum has the largest amplitude and copper has the least amplitude. The amplitudes of the cantilever over cadmium and aluminum are in between. In general, the amplitude of oscillation decreases with increasing electrical conductivity. The amplitude of oscillations, on the insulator is at least five times higher than that of the metals. Hence, it was not included in the figure for a direct comparison. The difference between the amplitude of oscillations between the insulator and the metallic samples is attributed to the generation of eddy currents in the metal. In an insulator, the magnetic field generated by the coil passes through the insulator without shielding. Hence, the entire magnetic field generated by the electromagnetic coil is sensed by the magnetic tip, producing large amplitude oscillations of the cantilever. On the other hand, in the presence of a metal, the oscillating electromagnetic field generates eddy currents in the metal shielding significant portion of the magnetic field that is sensed by the magnetic tip. The amplitude of oscillations of the cantilever on the metal is at least five times smaller than on insulator, because of the shielding effect.

The amplitude of the oscillations can be used to evaluate the eddy current force between the sample and the magnetic tip using Hooke's law. The spring constant of the cantilever is 0.1 $\mathrm{N} / \mathrm{m}$. However, since the cantilever was operated at the resonant frequency, the spring constant of the cantilever needs to be modified by the quality factor, $\mathrm{Q}$ of the cantilever to obtain dynamic spring constant. The dynamic spring constant was determined from the 
resonance curve for the cantilever using a method given in the literature (Sader, 1999). The eddy current force is calculated using the modified spring constant. In order to measure the eddy current force over a range of separation distance, the oscillation amplitude of the cantilever was measured at several fixed distances up to $550 \mathrm{~nm}$. The effect of the separation distance on the eddy current force is shown in Fig. 4.

The eddy current force decreases exponentially over distance and the force is large when the separation distance is less than $100 \mathrm{~nm}$. Above $100 \mathrm{~nm}$ separation, the force decreases rapidly and levels off after about $400 \mathrm{~nm}$. In metals with higher conductivity, the exponential decrease of the force is much more pronounced while in lower conductivity metals, the decrease in the force as a function of distance is gradual. The solid lines in Fig.4 indicate the exponential fit to the data. The behavior seen in Fig.4 is similar to the force curves studied in MFM (Murphy \& Spalding, 1999). The similarity is due to the fact that both MFM and eddy current force microscopy are functionally similar. The eddy current force distance curve is expected to follow inverse square law over the entire range of distance. Even in the force curves of MFM the inverse square behavior is not seen for all separation distances (Murphy \& Spalding, 1999). The reason for this behavior is the contribution of other forces in the distance ranges. The same argument holds true in the case of eddy current forces also. The inverse square law is observed up to a separation distance of $300 \mathrm{~nm}$. Beyond $500 \mathrm{~nm}$, the eddy current forces are weak and the amplitude is in thermal noise range.

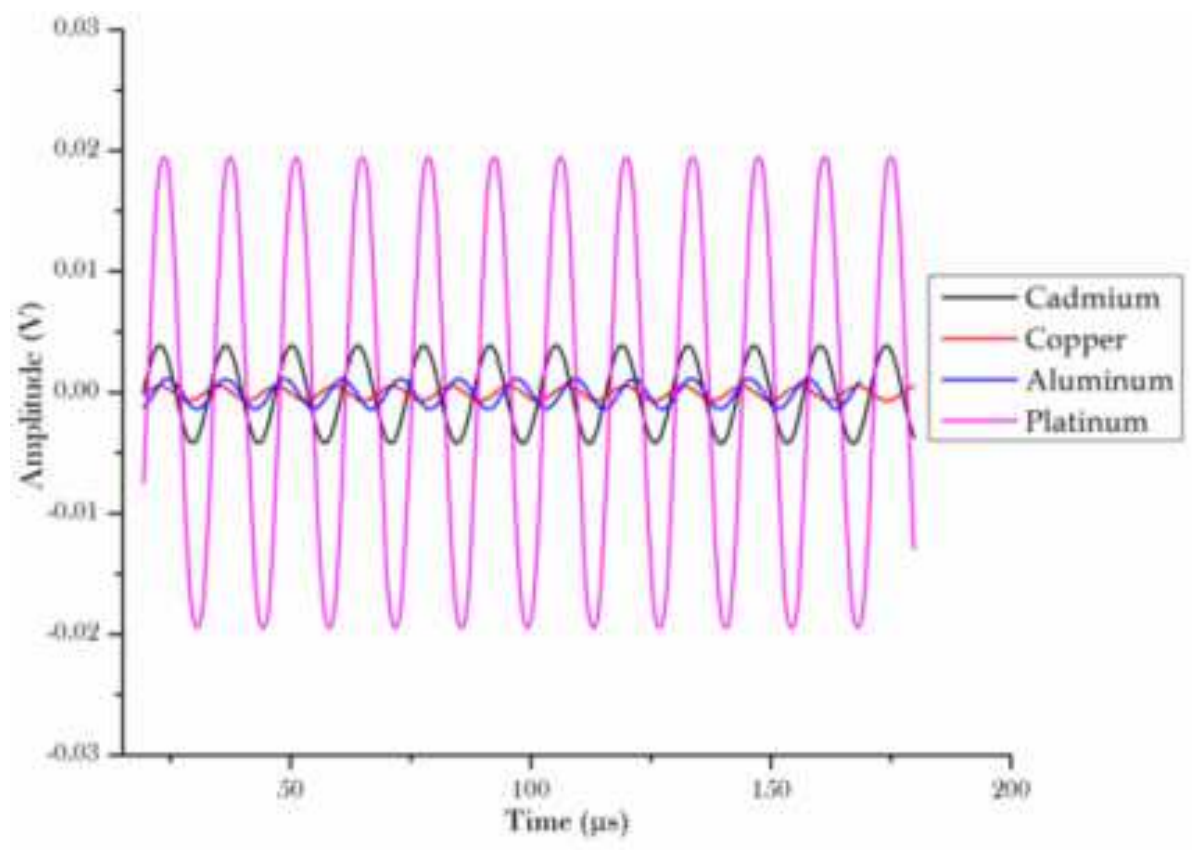

Fig. 3. Comparison of oscillation amplitudes of the AFM cantilever on different metallic samples at a separation distance of $50 \mathrm{~nm}$ and an excitation frequency of $82 \mathrm{kHz}$. The waveforms have been slightly shifted in time to show the waveforms separately. 


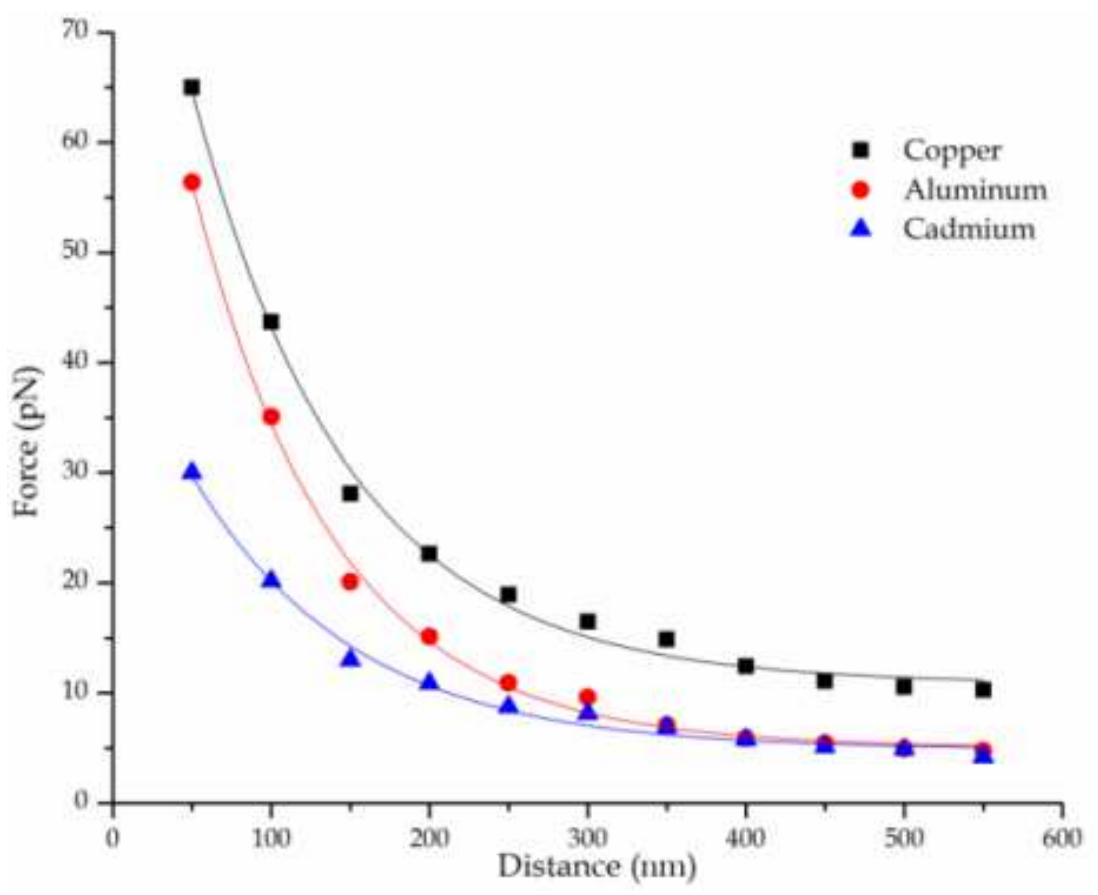

Fig. 4. Effect of separation distance between magnetic tip and sample surface on the eddy current force in different metals.

\subsection{Imaging electrical conductivity variations in bulk conductors}

It was shown that the force due to the eddy currents in a metal changes as a function of electrical conductivity. Therefore, by mapping the variations in the eddy current forces as the tip scans over the sample surface, one should be able to obtain an image of electrical conductivity. As the conductivity changes, the magnitude of the eddy current force changes and therefore, the image is a map of electrical conductivity variations. Before obtaining images, it is important to know the resonance spectra of the cantilever in order to achieve maximum sensitivity.A network analyzer (HP 8753D) was used to obtain the resonance characteristics of the cantilever coupled with the sample(Nalladega et al., 2008b). The resonance peaks of the cantilever while positioned over copper are shown in Fig. 5. The cantilever has resonance peaks at several frequencies, the dominant one being at $86 \mathrm{kHz}$ with other peaks at $280 \mathrm{kHz}, 508 \mathrm{kHz}$, and $580 \mathrm{kHz}$. While the images can be obtained at any of these frequencies, images obtained around $86 \mathrm{kHz}$ will have maximum contrast in the images due to the maximum amplitude at this frequency. Similar experiment was also done for titanium and the resonance peaks in this case were observed at $92 \mathrm{kHz}, 275 \mathrm{kHz}, 510$ $\mathrm{kHz}$ and $600 \mathrm{kHz}$. The resonance spectra of other metals (aluminum, cadmium) showed the peaks at similar frequencies. The differences in the resonant frequency can be attributed to many factors including thickness, conductivity, eddy current forces, penetration depth etc. (Siddoju et al., 2006). Therefore, the resonant characteristics of the cantilever should be characterized before obtaining an image. 


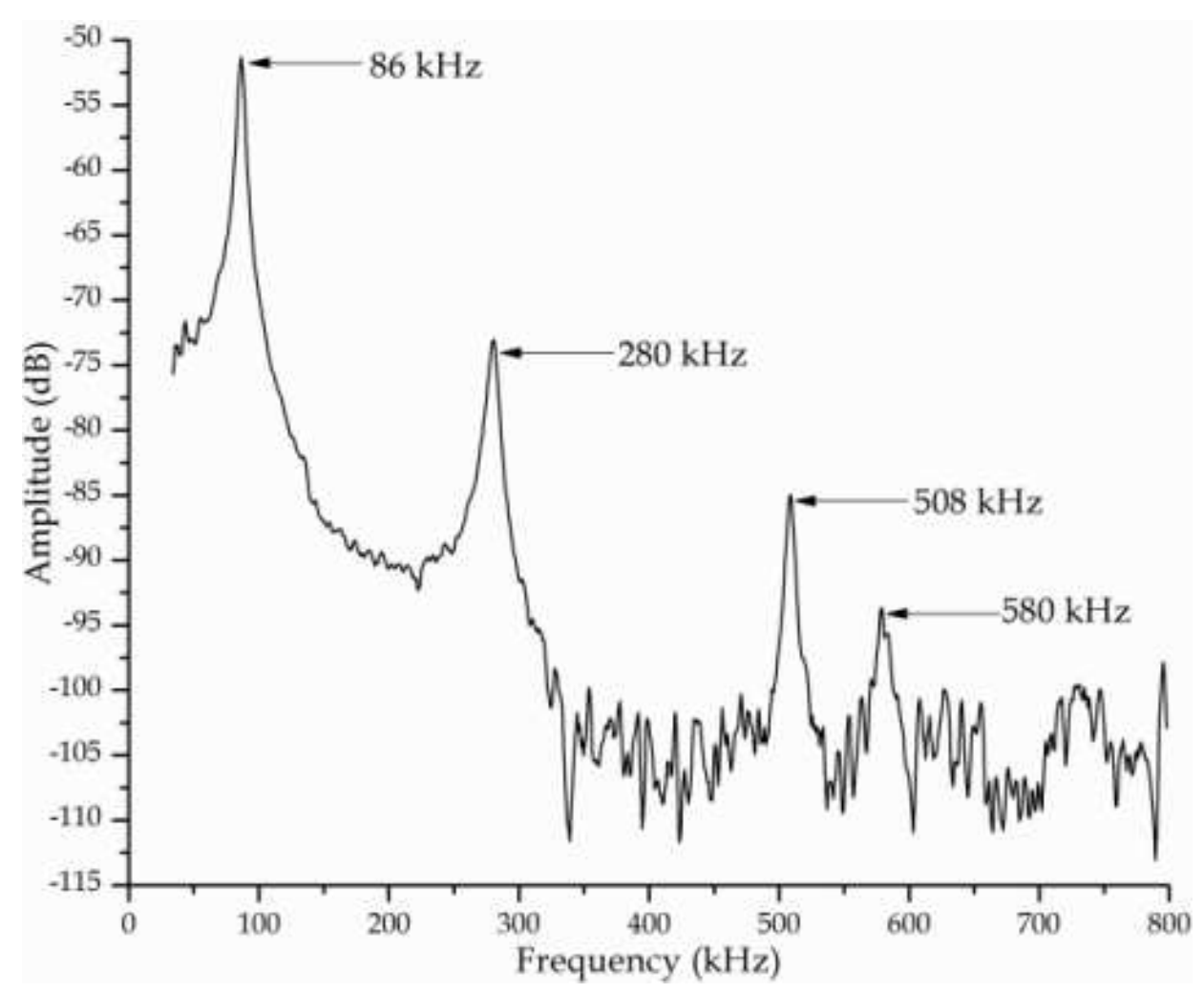

Fig. 5. Resonance spectra of the AFM cantilever positioned on copper showing multiple resonance peaks.

\subsubsection{Carbon fiber reinforced composite}

The eddy current imaging technique was first applied to image conductivity variations in a material with huge electrical conductivity variations. For this purpose, a carbon fiber composite with an average fiber diameter of $7 \mu \mathrm{m}$ was chosen (Nalladega et al., 2007). The electrical resistivity of carbon and the polymer matrix is $0.006 \Omega-\mathrm{cm}$ and $1 \times 1015 \Omega-\mathrm{cm}$ respectively. Figure 6 shows topography and electrical conductivity images of carbon fibers at an excitation frequency of $272 \mathrm{kHz}$ and a lift height of $50 \mathrm{~nm}$. The image on the left shows AFM topography and the image on the right is the eddy current image showing the electrical conductivity variations in the composite.

The contrast in the AFM image is due to variation of surface height and brighter regions indicate higher surface heights. Therefore, the carbon fibers appear bright in the image compared with the polymer matrix (Fig. 6a). Fig. $6 \mathrm{~b}$ shows the eddy current image of the same region. In the eddy current image the fibers appear dark while the polymer matrix appears bright. The difference in the contrast is due to the differences in the electrical conductivity of fiber and polymer. The matrix is almost an insulator and the magnetic field generated by the coil passes through without shielding and hence less damping of the cantilever, producing 


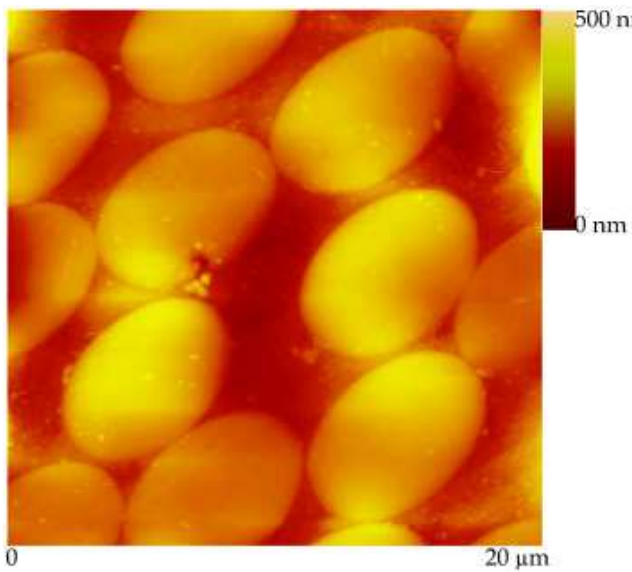

(a)

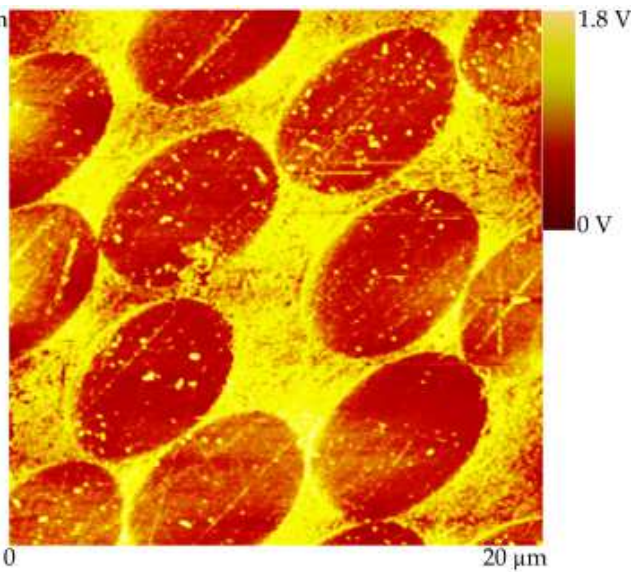

(b)

Fig. 6. (a) Surface topography and (b) eddy current images of carbon fibers reinforced in polymer matrix.

large amplitude of vibration of the tip-cantilever. Large amplitude produces significant output voltages from the photo-detector. When the tip is located on the carbon fiber, the magnetic field of eddy currents is shielded by the conductive fibers. Thus, vibration of the cantilever is dampened due to this shielding. Therefore,the magnetic tip-cantilever measures reduced amplitude compared with polymer matrix. Reduced amplitude of the cantilever produces lower output voltage from the photo-detector. Thus the carbon fiber appears darker than the polymer matrix in the eddy current image. Consequently, in eddy current images, darker contrast indicates higher conductivity regions and brighter contrast indicates less conductivity regions. The scale bar in the eddy current image represents the output voltage from the photodiode detector, which is proportional to the electrical conductivity.

A higher magnification conductivity image of the composite is shown in Fig.7. Along with the image a section analysis along the line shown in the image (Fig. 7b), is also presented. The profile above the center line represents the matrix and below the center line represents the fiber. The section analysis shows the variation of the amplitudes of the vibration of the cantilever as it scans the surface. The variation in amplitude is due to the variation in the magnetic field as the tip scans the surface. The strength of the magnetic field generated due to eddy currents is directly proportional to the electrical conductivity. Hence, the section analysis of the eddy current image shows the variations in relative conductivity. The section analysis shows that, at some regions there is a sharp transition in conductivity profiles at the boundary of matrix and fiber, while at other regions there is a gradual transition at the boundary, as shown by arrows in the image. In composite materials, it is very important for the proper bonding between the fiber and the matrix at the interface for maximum strength. Therefore, characterization of interface is important to estimate the strength of the composite. The image at the interface shows that high resolution imaging is possible using eddy current AFM. Thus, this technique is well suited for the characterization of the interface in composite materials based on the variations in electrical conductivity. 


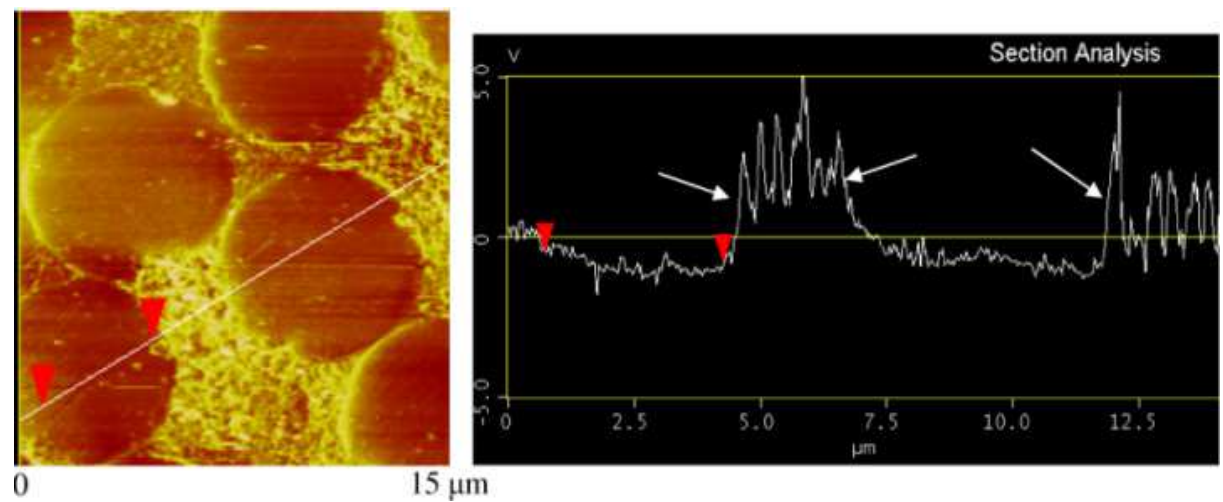

Fig. 7. A high resolution eddy current image of the carbon fibers and the section analysis along the line shown in the figure.

\subsubsection{Dual phase titanium alloy}

In electrically anisotropic metals, the electrical conductivity varies from grain to grain due to crystallographic orientation. An excellent example for the electrically anisotropic metallic alloy is a dual-phase titanium alloy, Ti-6Al-4V.Ti-6Al-4V alloys are used in high performance aerospace applications that require high toughness, good fatigue strength, and good corrosion resistance. Titanium exists in two crystal structures: hexagonal close packed ( $\alpha$-phase) and body centered cubic ( $\beta$-phase). The two phases often exist together in $\alpha-\beta$ alloys. The hexagonal phase has anisotropic electrical conductivity. In pure titanium the electrical resistivity in the basal plane is $45.35 \mu \Omega \mathrm{cm}$ and $48 \mu \Omega \mathrm{cm}$ in a plane normal to basal plane (Meaden, 1965). Thus grains with different crystallographic orientation will have different electrical conductivity. The electrical conductivity of the two phases is expected to be close. In order to test the feasibility of imaging small changes in conductivity using the eddy current AFM, the technique was applied to a dual phase Ti-6Al-4V sample (Nalladega, et al., 2008b). The microstructure of the alloy consists of circular primary $\alpha$ grains with a grain size of $10-20 \mu \mathrm{m}$ and fine lamellar $\alpha+\beta$ platelets.

Figure 8 shows surface topography and eddy current images on the Ti-6Al-4V sample. The image was obtained at an excitation frequency of $92 \mathrm{kHz}$ at a lift scan height of $50 \mathrm{~nm}$, between the tip and sample. From Fig. 4, it is evident that for higher sensitivity the separation distance should be less than $100 \mathrm{~nm}$. Therefore, the eddy current images shown in this chapter are obtained at a lift height of less than $100 \mathrm{~nm}$. The surface topography image shows $\alpha$ grains, the $(\alpha+\beta)$ grains and $\alpha$ platelets inside $(\alpha+\beta)$ grains with a maximum vertical height of $800 \mathrm{~nm}$ (Fig. $8 \mathrm{a})$. The $\beta$ between the $\alpha$ platelets cannot be observed clearly in the topography image. The eddy current force image (Fig. 8b) shows quite good contrast. The large $\alpha$ grains, the $(\alpha+\beta)$ grains and the $\alpha$ platelets inside $(\alpha+\beta)$ grains can be observed with significant contrast. Some of the large $\alpha$ grains that appear as a single grain in the AFM image appear to be consisting of smaller grains and $\alpha$ plates when viewed in the eddy current image. The difference in contrast in the eddy current imageis due to the difference in the electrical conductivities of $\alpha$ and $\beta$ phases. Since the electrical conductivities of the two phases are different, the vibration amplitudes of the cantilever and hence the eddy current forces will be different in the two 


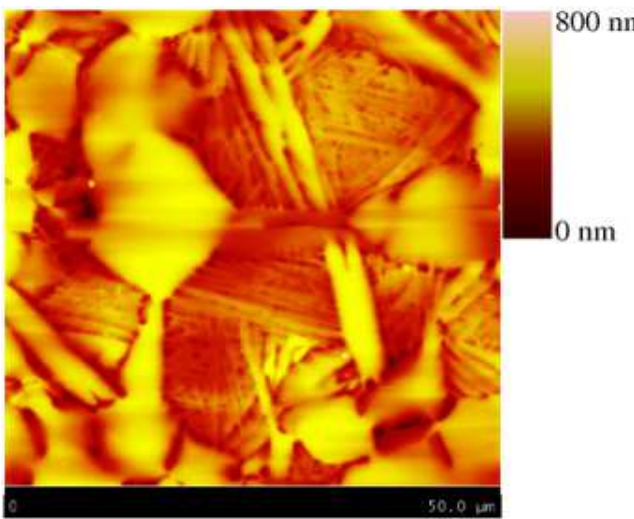

(a)

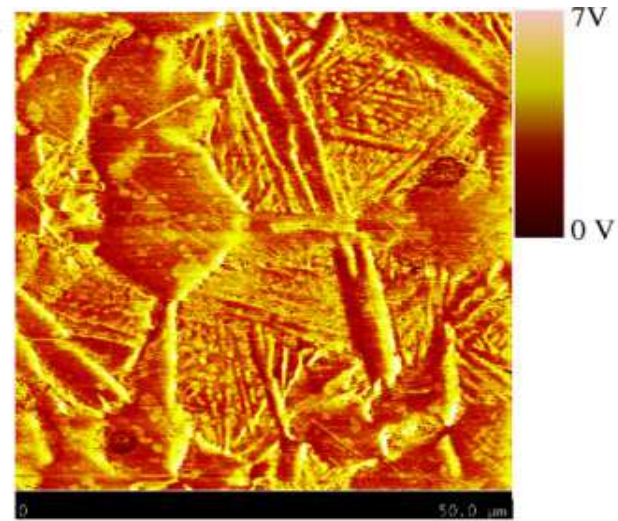

(b)

Fig. 8. (a) Surface topography and (b) eddy current AFM images of dual phase titanium alloy, Ti-6Al-4V (Nalladega et al., 2008b). (Reprinted with permission from American Institute of Physics)

phases. Consequently, the contrast shows bright to dark variations depending on the changes in conductivity.The lamellar structure can be clearly resolved in the eddy current image. Contrast among large $\alpha$ grains can also be observed in the eddy current image. Thus, the conductivity image shows significantly more structure than the topography image. The eddy current image on the titanium shows that the technique is sensitive to the small changes in the electrical conductivity of the material. The conductivity of the $\beta$ phase is different compared to $\alpha$ phase. The difference in the electrical conductivity between the two phases and the anisotropy in conductivity of the $\alpha$ phase enhances the contrast in eddy current images. These factors help in the observation of multiple grains, platelets in large $\alpha$ grains that appear to be single grain in AFM topography images. Although the surface roughness among the different phases and platelets are very small to show significant contrast in AFM images, the electrical conductivity is significantly different among different phases of the material and the anisotropy of electrical conductivity enhances the contrast in eddy current images.

A high magnification image of the platelets in the titanium alloy is presented in Fig. 9. A section analysis along the line shown in the images showing the variations in topography and conductivity respectively is also shown. In some regions it has needle like grains of alpha phase within a large grain. The surface topography image shows the needle like grains oriented in different directions (Fig.9a). The contrast is fuzzy and identifying individual platelets is difficult. On the other hand the eddy current image (Fig. 9b) shows the platelets with enhanced contrast at the boundary. The contrast at the grain boundary is quite strong because of significant change in the conductivity and due to modification of the eddy currents near the boundary. The conductivity is modified near the grain boundaries due to extra scattering of the electrons. The widths of the dark and bright phases shown in the line scan of the eddy current image are $560 \mathrm{~nm}$ and $520 \mathrm{~nm}$ respectively.

The eddy currents diffuse from the bottom of the sample through the thickness of the sample. Hence, the eddy current force is an average over the thickness of the sample. If the electrical conductivity is inhomogeneous in the thickness direction, the eddy current force is an average 


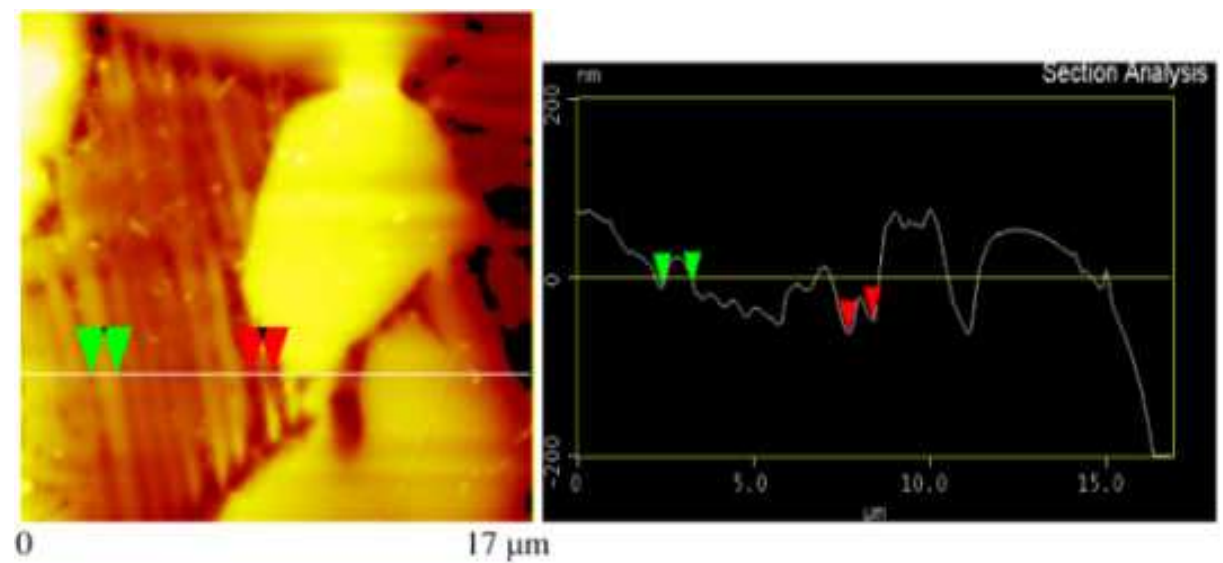

(a)
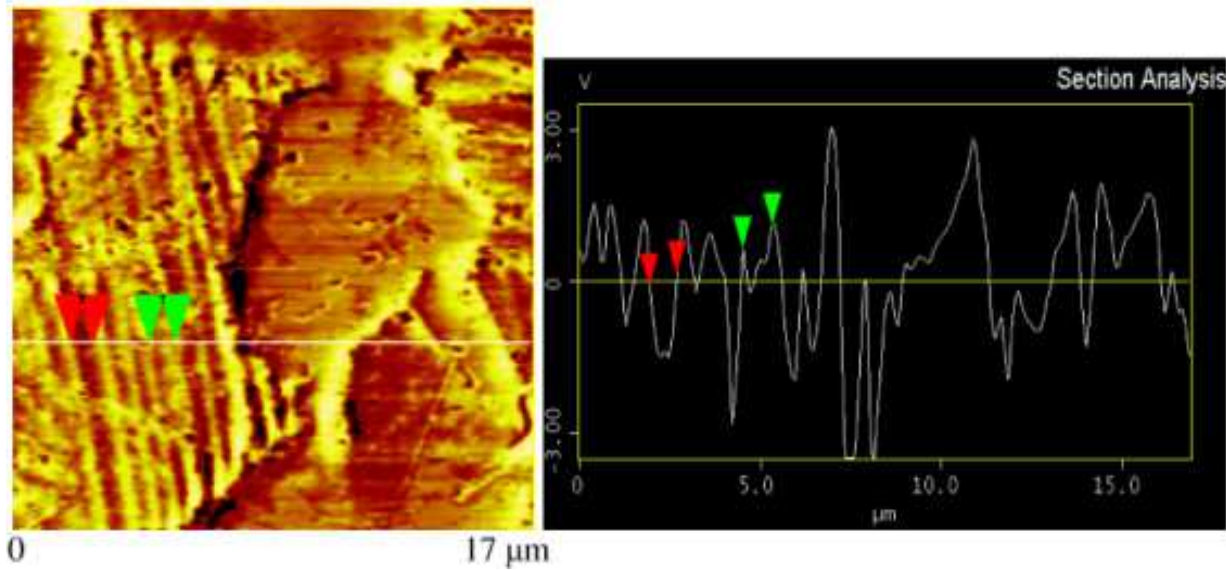

(b)

Fig. 9. (a) Surface topography and the corresponding line scan across the line shown in the topography image.(b) Eddy current image and the corresponding line scan across the line shown in the eddy current image of dual-phase titanium alloy.

over the sample thickness. While this appears to be a limitation of the technique, it may be useful in imaging subsurface defects or cracks that cause inhomogeneities in electrical conductivity. In fact this has been effectively used in eddy current NDE(Huang et al., 2006).

\section{Spatial resolution of the eddy current AFM}

A carbon nanofiber reinforced composite was used to determine the spatial resolution of the eddy current AFM (Nalladega et al., 2008b). The diameters of the fibers are in the range of 20-100 nm and the length is known to be in the range of fraction of microns to tens of microns. Figure 10 shows the topography and eddy current images of the carbon nanofibers, together with section analysis along a single fiber, as shown in the figure. The images were 


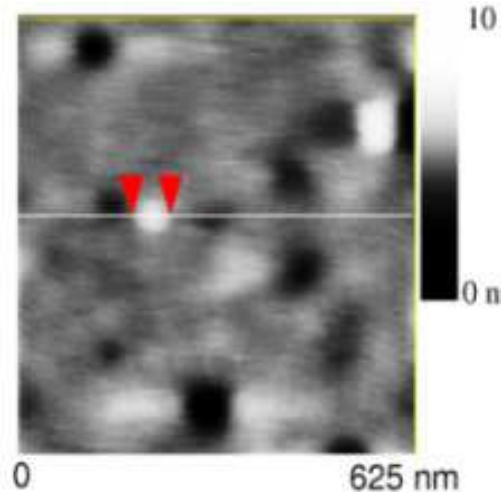

0

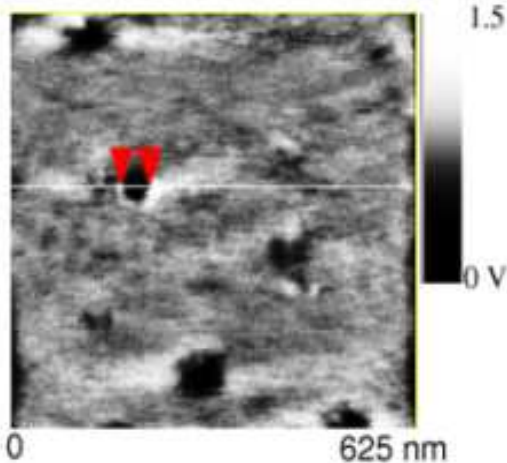

$625 \mathrm{~nm}$

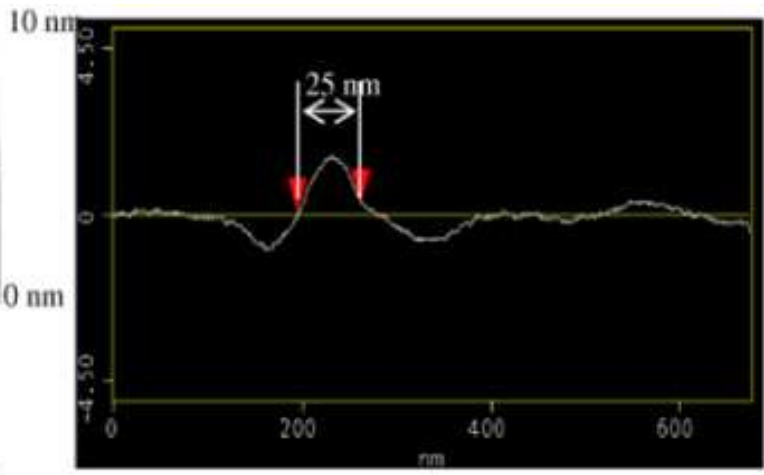

(a)

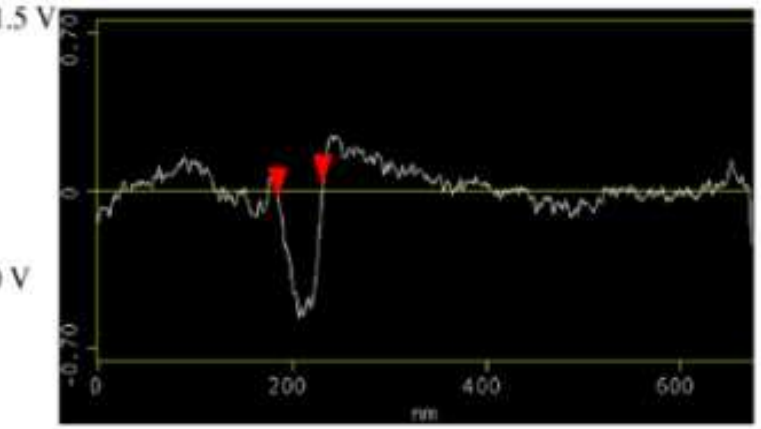

(b)

Fig. 10. (a) Surface topography and (b) eddy current images of carbon nanofibers (Nalladega et al., 2008b). (Reprinted with permission from American Institute of Physics).

obtained at an excitation frequency of $85 \mathrm{KHz}$ and a lift height of $50 \mathrm{~nm}$. A $25 \mathrm{~nm}$ diameter nanofiber can be resolved in eddy current images. In the experiment, a magnetic tip with a nominal diameter of $20 \mathrm{~nm}$ was used for imaging. The magnetic field sensed by the tip is larger than the diameter of the tip. The spatial resolution in AFM depends on the diameter of the probe and interaction forces between the tip and the sample (Banerjee et al., 1996; Hutter \& Bechhoefer, 1993a). Long range tip-sample interaction forces degrade the resolution of the AFM (Goodman \& Garcia, 1991; Hutter \& Bechhoefer, 1993b). Since the magnetic forces due to the eddy currents are long range interaction forces and the magnetic tip interacts with a volume of magnetic field larger than the tip diameter, the resolution is slightly larger than the tip diameter.

\section{Low-frequency electromagnetic properties of nanostructured materials}

The reduction of the size of metals results in significant changes in the electromagnetic properties compared with the bulk metals. In particular, the electromagnetic response of metallic nanoparticles is the subject of many research efforts during the last decade (Kelly et 
al., 2003; Michaels et al., 1999; Tominaga et al., 2001). The metallic nanoparticles show plasmon resonance when subjected to electromagnetic fields. Plasmon resonance occurs due to coherent oscillation of the conduction band electrons induced by the incident EM field at opticalfrequencies. The surface plasmon resonance in metallic nanoparticles is usually characterized using near-field optical techniques such as scanning near-field optical microscopy (SNOM) (Courjon, 2003; Okamoto, 2006). The plasmon resonances in the metallic nanoparticles occur in the optical frequency range and they result in powerful localized sources of electric field. In these techniques, the electric field around the nanoparticle is imaged.

While the electromagnetic properties of nanoparticles have been extensively studied at optical frequencies, little information is available on the low frequency (less than $1 \mathrm{MHz}$ ) interaction of electromagnetic waves with metallic nanoparticles. It is known that in the presence of an external magnetic field, low frequency electromagnetic waves can propagate as helicons in metals (Maxfield, 1969; Petrashov, 1984). Helicons are circularly polarized electromagnetic waves that propagate with very low phase velocity. When the mean free path of electrons is sufficiently large and the frequency $\omega$ of the wave is low enough, the electrons affected by the Lorentz force would drift in the direction perpendicular to the plane formed by the uniform magnetic field and the electric field of the wave. Helicon resonance modes in metals can be observed whenever the helicon frequency approaches the cyclotron frequency, i.e. $\omega \rightarrow \omega_{\mathrm{c}}$. The cyclotron frequency $\omega_{\mathrm{c}}$, is given by

$$
\omega_{c}=\frac{e B_{o}}{m^{*} c}
$$

where $e$ is the electron charge, $\mathbf{B}_{0}$ is the static magnetic field, $m^{*}$ is the effective mass of the electron and $c$ is the speed of light. In addition, the local conditions, viz., $\omega \tau<<1$, and $k l<<1$ have to be satisfied. Here, $\omega$ is the excitation frequency, $\tau$ is the relaxation time of the electrons, $k=2 \pi / \lambda$ is the helicon wave vector and $l$ is the electron mean free path. The local conditions are fulfilled for the frequencies $\omega<10^{8} \mathrm{~s}^{-1}$. The phase velocity of helicons is smaller than the speed of the light. At typical metallic densities, the plasma frequency, $\omega_{\mathrm{p}}$ $=10^{16} \mathrm{sec}^{-1}$; for a frequency $\omega=10^{7} \mathrm{sec}^{-1}$ and $\omega_{\mathrm{c}}=10^{11} \mathrm{sec}^{-1}$, the phase velocity of a helicon is approximately $30 \mathrm{~m} / \mathrm{s}$. The magnetic field of helicon wave is much larger than the magnetic field of an ordinary electromagnetic wave with the same electric field.

Helicon wave propagation and its resonance have been observed in high purity metals only at very low temperatures (Bowers, 1961; Chambers, 1962; Houck, 1964; Taylor, 1963). At very low temperatures, the electron mean free path of most metals is in mm range. To observe helicon resonances, an electromagnetic coil is excited with frequency in $\mathrm{MHz}$ range. The sample and the coil are placed in a static magnetic field of 1-100 kG and cooled to liquid helium temperature. The impedance of the coil is measured as the magnetic field is varied. Helicon resonance modes in metals can be observed when the excitation frequency approaches the cyclotron frequency. In a typical metal placed in an external static magnetic field of $1 \mathrm{kG}$, the cyclotron frequency is about $10 \mathrm{MHz}$.

The electron mean free path of gold at room temperature is about $50 \mathrm{~nm}$. However, the cyclotron resonance frequency at a magnetic field of $1 \mathrm{kG}$ is approximately $10^{6}-10^{7} \mathrm{~Hz}$. To observe helicon resonances at room temperature the electromagnetic frequencies have to be in the range of $10-100 \mathrm{MHz}$. Eq. (17) shows that helicon wave propagation at low 
frequencies in metallic nanoparticles can occur if either the effective mass is large or the static magnetic field is extremely high.

It was shown that the effective mass of electrons can be dramatically increased in artificial metallic lattice structures made of thin metallic wires (Pendry et al, 1996). When the metallic structure was subjected to an electromagnetic field, it has been shown that the effective mass of the electron increased by four orders of magnitude. By confining electrons to thin wires, an enhancement of their mass was achieved by 4 orders of magnitude. Consequently, the plasma frequency was reduced to $\mathrm{GHz}$ range from $\mathrm{UV}$ or optical frequency ranges. Following similar arguments, for a periodic array of metallic nanoparticles of gold or silver with diameter of 200 $\mathrm{nm}$, and $1 \mu \mathrm{m}$ spacing, it can be shown that the effective mass of the electron increases by two orders of magnitude and the effective electron density decreases by three orders of magnitude. The cyclotron resonance frequency was found to be few hundred $\mathrm{kHz}$ in a magnetic field of 1 $\mathrm{kG}$, and the phase velocity of helicons was less than $100 \mathrm{~mm} / \mathrm{s}$. Thus it is possible to generate helicon waves and resonances in metallic structures at very low frequencies at room temperatures whenever these conditions are satisfied.

The eddy current AFM system was used to detect and image helicon resonances in a distribution of metallic nanoparticles (Nalladega et al., 2011). Nanoparticles of platinum were deposited on a glass substrate using a process known as Through Thin Film Ablation (TTFA)(Murray \& Shin, 2008). The target consisted of $20 \mathrm{~nm}$ thin film of Pt that was sputter deposited onto a UV transparent fused silica. The target was then ablated through the silica support at an energy density of $0.5-1.0 \mathrm{~J} / \mathrm{cm}^{2}$. The nanoparticles are synthesized without agglomeration and have a uniform size distribution. Figure 11 shows a SEM micrograph of platinum nanoparticles synthesized using TTFA.

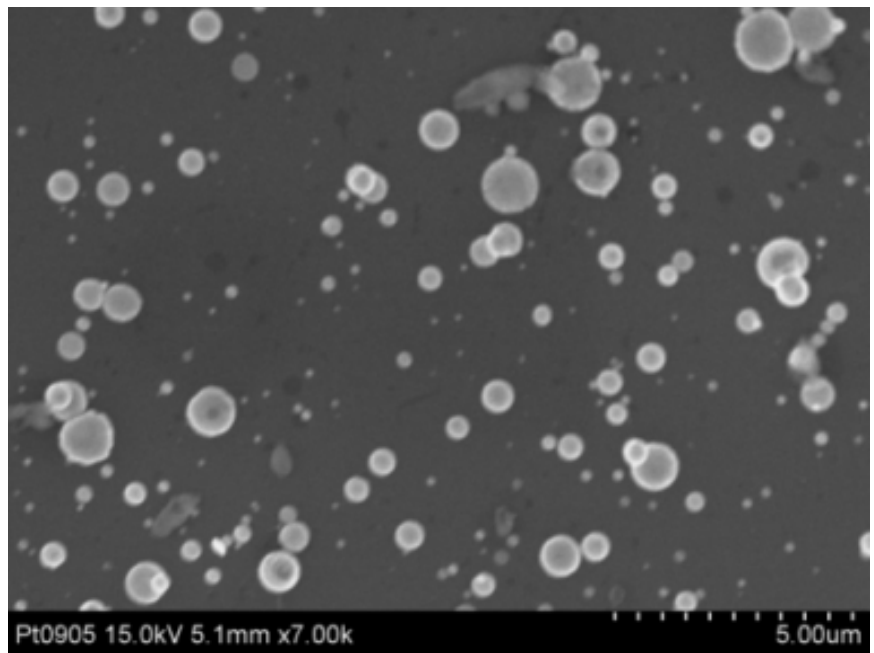

Fig. 11. Distribution of the platinum nanoparticles synthesized using through thin film ablation process.

Figure 12 shows surface topography and the eddy current images of individual platinum nanoparticles obtained at $90 \mathrm{kHz}$. Fig. 12a shows the surface topography and eddy current 


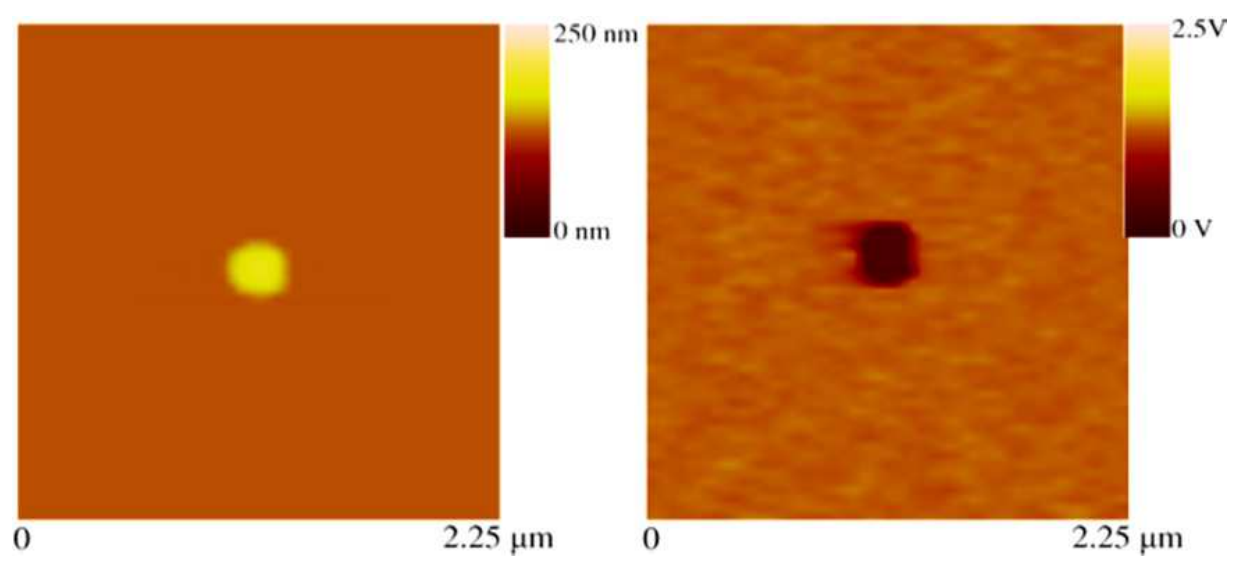

(a)

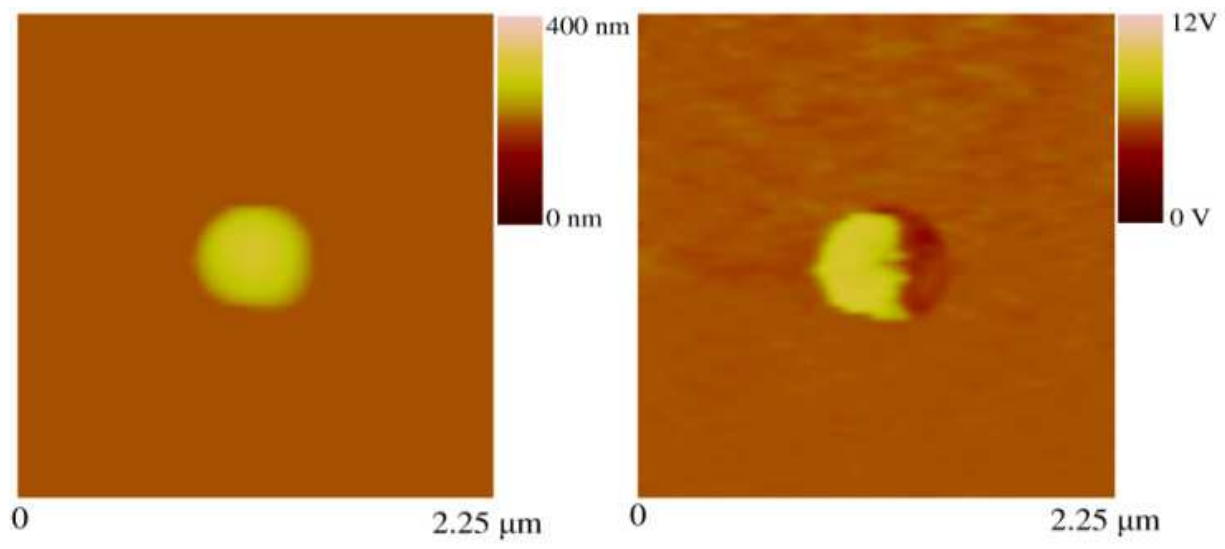

(b)

Fig. 12. Surface topographyand eddy current images of Pt nanoparticles (Nalladega et al., 2011). (Reprinted with permission from American Institute of Physics)

images of a $250 \mathrm{~nm}$ diameter nanoparticle. The eddy current image shows a darker contrast varying uniformly across the nanoparticle. Figure $12 \mathrm{~b}$ shows another nanoparticle with 500 $\mathrm{nm}$ diameter at the same frequency. The surface topography image is similar to the $250 \mathrm{~nm}$ particle but the contrast in the eddy current image is very different. The image shows a particle with half bright and half dark regions. The contrast in eddy current AFM is based on the electrical conductivity. Thus, it appears there is a variation in electrical conductivity within the particle. However, it is extremely unlikely that a single particle can have two different conductivities especially if the particle is made of single material. Figure 13 shows the topography and eddy current images of platinum nanoparticles at a different region of the sample at $90 \mathrm{kHz}$. The images show three platinum nanoparticles of different size. Again, the eddy current image distinctly shows different contrast among the nanoparticles. Two of the nanoparticles are seen with a contrast similar to that of Fig.12b. A nanoparticle with $800 \mathrm{~nm}$ diameter and a dark region between two bright regions can also be seen in the image. 


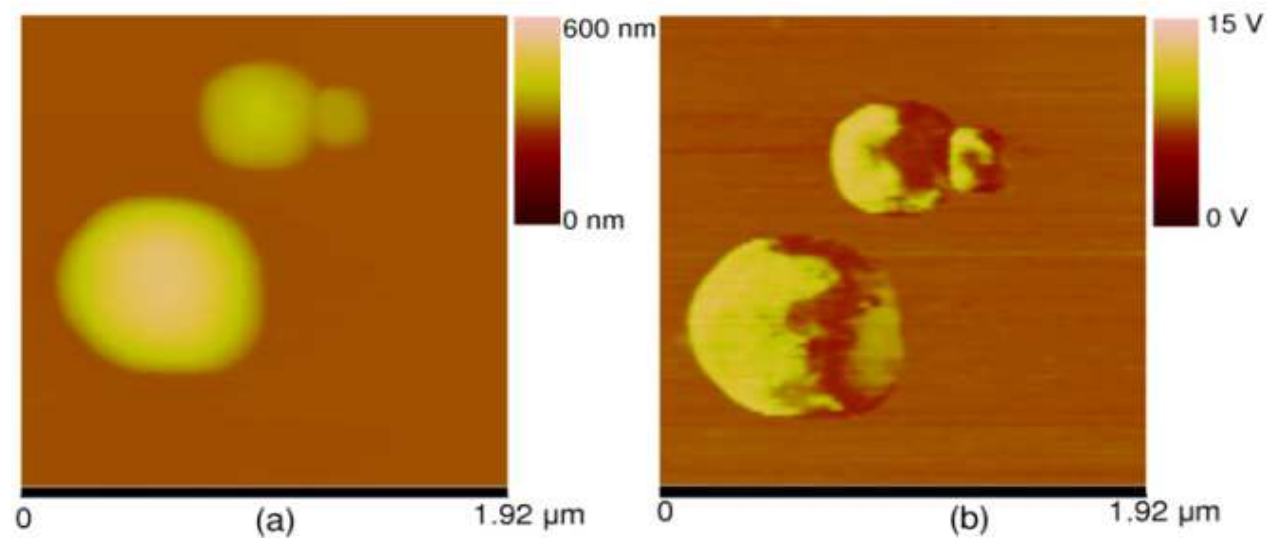

Fig. 13. (a) Surface topography and (b) eddy current images of platinum nanoparticles. The $800 \mathrm{~nm}$ Pt particle is split into three parts showing bright-dark-bright contrast within the particle (Nalladega et al., 2011). (Reprinted with permission from American Institute of Physics)

The eddy current images shown in Fig.12 and 13 are similar to the surface plasmon resonance images on spherical metallic nanoparticles reported in the literature (Kelly et al., 2003; Michaels et al., 1999; Tominaga et al., 2001;Courjon, 2003; Okamoto, 2006). Based on the similarity of the plasmon resonance images of nanoparticles with the images shown in Fig.12 and 13, it appears the images show the resonance behavior of the nanoparticles at low frequencies. While the optical techniques image the electric field around the nanoparticle, the eddy current AFM images the magnetic field around the nanoparticle. Therefore, we believe the source of the contrast seen in the above images is due to the resonances of low-frequency electromagnetic waves like helicons. This is further reinforced by the decrease in plasmon frequencies of artificial nanostructures (Pendry et al, 1996). Since a sharp magnetic probe is used instead of a pick-up coil to detect the helicons, it is now possible to image helicon resonance modes with nanometer resolution.

The magnetic field images of the nanoparticles can be compared with the magnetic lines of force of spherical particles subjected to electromagnetic radiation. The magnetic lines of force of spherical particles for different resonance modes are schematically shown in Fig.14. Higher order resonances have been observed in bigger particles in the sample showing regions of bright and dark band contrast. The shapes of the resonances have been observed to change with the diameter as well as the shape of the particle. The contrast can be explained based on accommodating tiny magnetic inside the particle with alternating the magnetic poles such that no two adjacent magnetic poles are alike.

Although the helicon resonance calculations assume periodic arrangement of square array, it was shown that for significant decrease of plasmon resonance frequency the actual lattice geometry may not be significant (Pendry et al., 1996). The experimental configuration developed in this work allows visualizing the helicon resonances in individual members of the structure with varying dimensions of the metallic nanoparticles and interparticle spacing. 

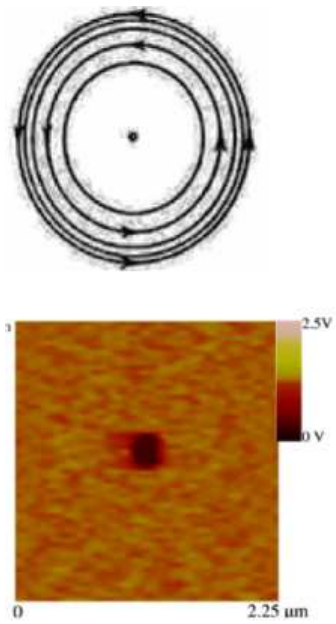
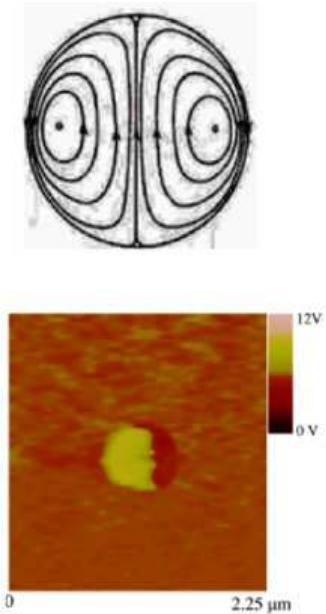
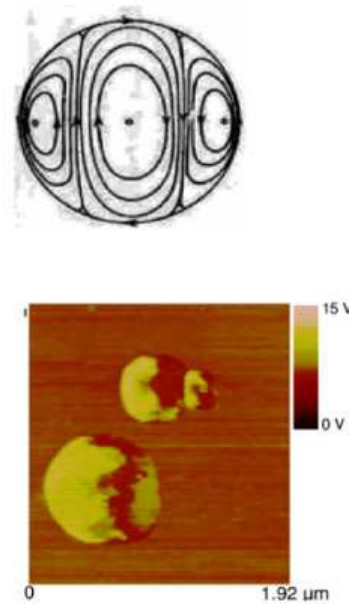

Fig. 14. Magnetic lines of force for the first, second, and third resonance modes in spherical particles and the correspondingmagnetic field images of Pt nanoparticles (Nalladega et al., 2011). (Reprinted with permission from American Institute of Physics)

\section{Characterization of magnetic properties}

In MFM, a ferromagnetic probe is attached the cantilever and scanned across a ferromagnetic sample to measure the magnetic interactions between the tip and the sample. For soft magnetic samples, the magnetization of the sample will be affected by the stray magnetic field of the tip. Therefore, the interpretation of the magnetic contrast in MFM images for such samples is difficult.This has led to the characterization of magnetic properties without a magnetic probe (Hoffmann et al., 1998). The technique uses a vibrating non-magnetic conducting tip to image the magnetic domains of a ferromagnetic sample. The oscillating conducting tip induces currents within the tip which is dependent on the magnetic properties of the sample.Experimental techniques to image magnetostrictive properties of magnetic materials using AFM have also been reported in the literature (Berger et al., 2000; Wittborn et al., 2000; Polushkin et al., 2003).

When a magnetic field is applied to a magnetic material, strains are generated in the material. This phenomenon is known as magnetostriction. When an oscillating magnetic field is applied to a ferromagnetic material, magnetostrictive strains are induced based on the frequency of the excitation. The magnetostrictive strain does not change the sign when the direction of the field is reversed. Therefore, the material produces strains at twice the excitation frequency. The strain due to magnetostriction is dependent on the magnetization of the domains and provides information about the magnetoelastic properties. Therefore, magnetostriction can be used to study the magnetic properties.The eddy current AFM system has been modified to image magnetic properties with a nonmagnetic probe (Nalladega et al, 2009). The approach is based on the measurement of strains due to magnetostriction in magnetic materials. Since the strains produced are a function of magnetization of individual domains, this technique can be used to image magnetic domains without the need of a magnetic probe. The technique is used to image differences 
in magnetostriction in amorphous and nanocrystalline magnetic melt spun ribbons. The contrast is explained based on the magnetostrictive interactions between a nonmagneticprobe and ferromagnetic material.

Amorphous and nanocrystalline magnetic ribbons of the nominal composition $\mathrm{FeSiBNbCu}$ alloy were used for this purpose. These materials are also known as FINEMET alloys (Yoshizawa et al., 1988; McHenry et al., 2003; Herzer, 1993). The nanocrystalline magnetic ribbon of FINEMET alloy is composed of nanosized crystallites embedded in a residual amorphous matrix. The nanocrystalline phase exhibit exceptionally soft magnetic properties which arise from a very small magnetostriction (Slawska-Waniewska \& Lachowicz, 2003). An AC magnetic field with a frequency $f=58 \mathrm{kHz}$ was applied to the sample. Since magnetostriction is proportional to $2 f$, the excitation frequency was chosen such that the $2 f$ frequency is close to the resonant frequency of the cantilever in contact with the sample. A contact mode nonmagnetic AFM tip was scanned across the sample in contact mode. The magnetostrictive strains cause the cantilever to oscillate at $2 f$ frequency. The amplitude of vibration of the cantilever at $2 f$ frequency is detected by the lock-in amplifier to obtain topography and magnetostriction images simultaneously.

Figure 15 shows the topography and magnetostriction images on the amorphous sample. The surface topography image (Fig.15a) shows a uniform contrast with no visible features. However the magnetostrictive image (Fig. 15b) shows circular bright and dark regions within the scan area. The bright regions correspond to larger amplitudes of cantilever vibration and consequently higher strains. The dark regions indicate that the amplitudes of the cantilever vibration are small and thus correspond to relatively lower strains due to magnetostriction. Therefore, the image shows that there is a variation in the local magnetostriction. The amorphous magnetic ribbons exhibit higher saturation magnetostriction. This is confirmed by the brighter regions in the magnetostriction image. Since magnetostrictive strain is a function of the magnetization of each domain, the magnetostriction image can be used to identify magnetic domains. The bright and dark regions represent magnetic domains (Takata \& Tomiyama, 2000), as shown by arrows in Fig. 15b.
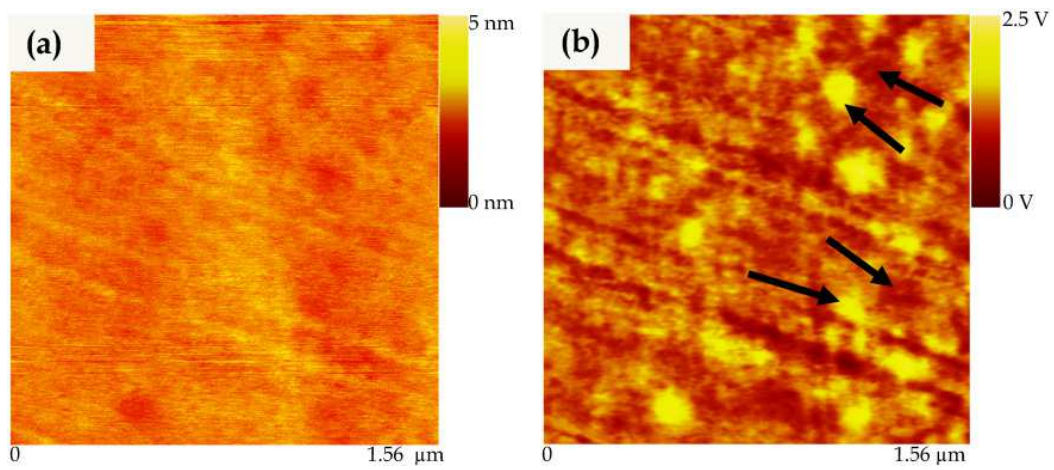

Fig. 15. (a) Surface topography and (b) magnetostriction image of amorphous magnetic ribbon sample. Some of the domains are indicated by arrows.

Figure 16 shows the surface topography and the magnetostriction images of the nanocrystalline sample. The images show the formation of crystals due to the annealing of 
amorphous sample with the size of the nanocrystals ranging from $50 \mathrm{~nm}-100 \mathrm{~nm}$. The topography image (Fig. 16a) shows the nanocrystals embedded in amorphous matrix with a maximum vertical height of $200 \mathrm{~nm}$. In the magnetostriction image (Fig.16b), most of the nanocrystalline phase appears with dark indicating the magnetostriction is smaller compared to that of amorphous phase. However, the presence of dark and bright regions locally can be observed. This indicates the presence of a small effective saturated magnetostriction. Figure 17 shows a higher magnification image of the nanocrystalline phase of the sample. Brighter regions within a domain can be observed in the magnetostriction image (Fig.17b). This indicates the saturation of magnetostriction in the domain. This results in a deformation, dependent on the magnetization direction of the domain (Takata \& Tomiyama, 2000). At the domain wall the magnetic moments changes direction, resulting in the deformation of material. The magnetization of the sample is inplane and the external magnetic field is perpendicular to the plane. Therefore each of the domains is subjected to rotational forces and consequently the amplitude of AFM cantilever is large near the domain walls. Based on this interpretation, the brighter region shown by the arrow (Fig. 17b) is a domain wall with a width of $40 \mathrm{~nm}$.
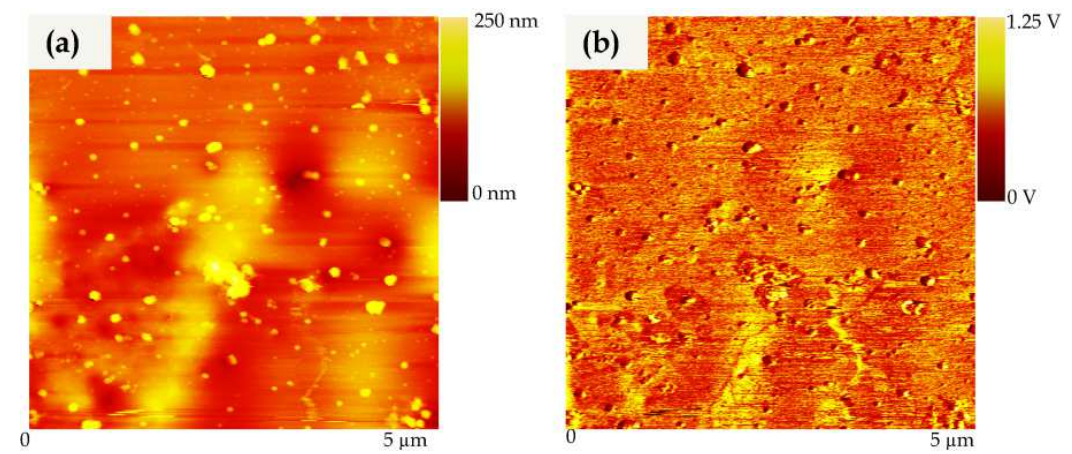

Fig. 16. (a) Surface topography and (b) magneto-elastic images of nanocrystalline magnetic ribbon sample. Nanocrystalline phase can be observed in the images.
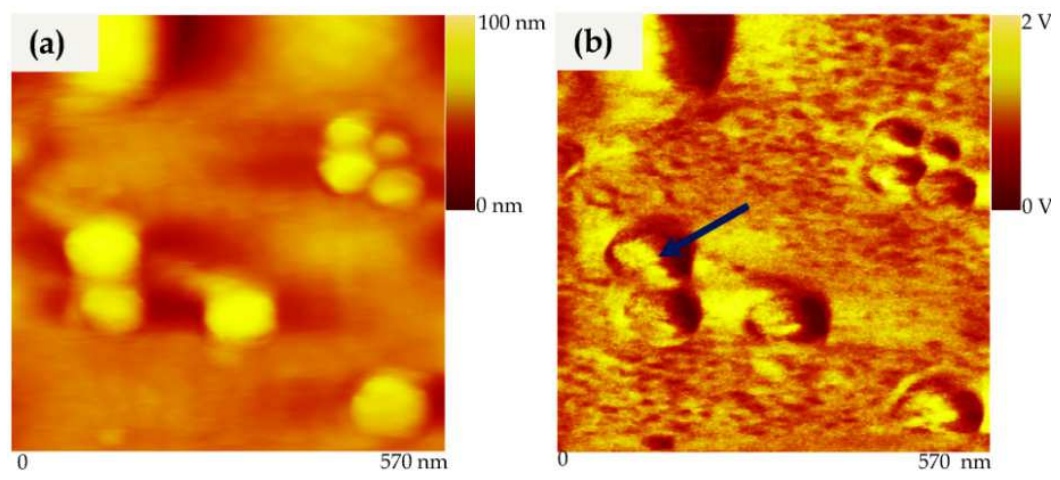

Fig. 17. A high magnification (a) surface topography and (b) magneto-elastic images of the nanocrystalline FINEMET alloy magnetic ribbon. A domain wall with a width of $40 \mathrm{~nm}$ is shown by arrow. 


\section{Conclusion}

A new AFM based method to measure and image electrical properties of material with nanometer resolution and high sensitivity is presented. The technique is based on generating eddy currents in conductors and using a magnetic tip to image the magnetic field due to eddy currents. The magnetic fields generated are a function of the electrical conductivity of the sample. Thus the image is a representation of the electrical conductivity. A cantilever with a spring constant of $0.1 \mathrm{~N} / \mathrm{m}$ was selected for the experiments for maximum sensitivity. Eddy currents in the samples were generated by a small electromagnetic coil. An external electronic instrumentation module was developed to generate images of both surface topography and electrical properties simultaneously at a given location on the sample. The electrical conductivity measurements are obtained in noncontact mode. Eddy current forces were experimentally measured in different metals. It was shown that the magnitude of the eddy current forces in the metal is related to its electrical conductivity. The effect of separation distance between the tip and the sample surface was also studied. It was shown that for maximum sensitivity the tip-sample separation should be less than $100 \mathrm{~nm}$.

The technique was used to image carbon fibers reinforced in a polymer matrix. The contrast in the images is explained based on the huge electrical conductivity variation between the fibers and the matrix. Next, small variations in electrical conductivity variations in a dual-phase titanium alloy, Ti-6Al-4V were imaged. The difference in electrical conductivity from $a$ and $\beta$ phases of the alloy is small. Also, the difference in the electrical conductivity of the HCP phase is around 6\%. The eddy current AFM was able to distinguish between two phases based on electrical conductivity variations. Contrast difference from grain to grain was also observed. The spatial resolution of the system was found to be approximately $25 \mathrm{~nm}$.

In addition to the electrical characterization, the technique was also used to characterize magnetic and electromagnetic properties at the nanoscale with minor modifications. The low-frequency electromagnetic interaction with metallic nanoparticles was studied for the first time using the technique. It was demonstrated that it is possible to generate and image helicon wave resonances in metallic nanostructures with nanometer resolution. The same methodology was modified slightly to image magnetic properties using a nonmagnetic probe in contact mode. The approach was based on magnetostrictive strains in ferromagnetic samples subjected to alternating magnetic field. Magnetostriction images were obtained on amorphous and nanocrystalline magnetic ribbon samples. The images show that it is possible to image magnetic domains without a magnetic probe.

The eddy current AFM combines the localized nature and high sensitivity of eddy currents and high spatial resolution and the versatility of AFM. Since induced currents are used in this method, no bias voltage is required to be applied between the sample and the tip. This also means that conductive tips are not needed for conductivity imaging. The electrical conductivity images are obtained in non-contact mode.For higher sensitivity to electrical conductivity variations, flexible cantilevers are used. Since a coil is used to generate eddy currents, eddy current densities can be independently adjusted for different materials based on electrical conductivity of the sample. The vibration spectra of the cantilever over the 
sample surface showed that multiple resonance peaks are present. Thus the technique can be used to obtain conductivity images multiple frequencies. This facilitates the study of the dependence of frequency on the contrast observed in eddy current images. The same instrumentation can be used for characterization of multiple material properties with some modifications. Finally, since eddy currents are conventionally used in nondestructive evaluation of defects, the methodology can also be used to perform micro/nano NDE to detect nanoscale defects.

\section{Acknowledgment}

One of the authors (V.Nalladega) would like to acknowledge the financial support of Dayton Area Graduate Studies Institute in completion of the work. He also acknowledges the support and encouragement of Dr. Allan Crasto, Associate Director of University of Dayton Research Institute. The authors would also like to acknowledge the financial support from Wright-Patterson Air Force Base. The authors would like to thank Dr. Terry Murray (UDRI) for the platinum nanoparticles sample and Drs. Mike Gigliotti and P. R. Subramanian (GE Global Research) for providing the amorphous and nanocrystalline magnetic ribbon samples.

\section{References}

Banerjee, S.; Sanyal, M. K.\& Datta, A. (1996). A Simulation Study of Multi-Atom Tips and Estimation of Resolution in Atomic Force Microscopy. Applied Surface Science, Vol.99, No.3, pp. 255-260

Berger, R.; Krause, F.; Dietzel, A.; Seo, J. W.; Fompeyrine, J. \& Locquet, J.-P. (2000). Nanoscale Magnetostrictive Response in a Thin Film Owing to a Local Magnetic Field. Appl. Phys. Lett., Vol.76, No.5, pp. 616-618

Betzig, E.; Trautman, J. K.; Harris, T. D.; Weiner, J. S.\& Kostelak, R. L. (1991). Breaking the Diffraction Barrier: Optical Microscopy on a Nanometric Scale. Science,Vol.251, No.5000, pp. 1468-1470

Binnig, G.; Rohrer, H. \& Gerber, Ch. (1986). Atomic Force Microscopy. Phys. Rev. Lett., Vol.56, No.9, pp. 930-933

Bluhm, H.; Wadas, A.; Wiesendanger, R.; Roshko, A.; Aust, J. A.\& Nam, D. (1997). Imaging of Domain-inverted Gratings in $\mathrm{LiNbO}_{3}$ by Electrostatic Force Microscopy. Appl. Phys. Lett., Vol. 71, No.1, pp. 146-148

Born, M.\&Wolf, E. (1999). Principles of Optics, Cambridge University Press,Cambridge, UK

Bowers, R.; Legendy, C.\& Rose, F. 1961. Oscillatory Galvanomagnetic Effect in Metallic Sodium. Phys. Rev. Lett., Vol.7, No.9, pp. 339-41

Burnham, N.; Kulik, A. J.; Gremaud, G.; Gallo, P. J. \& Oulevey, F. (1995). Scanning Local Acceleration Microscopy. J. Vac. Sci. Technol., Vol. 14, No.2, pp. $749-799$

Chambers, R. G. \& Jones, B. K. (1962). Measurement of High-Field Hall Effect by an Inductive Method. Proc. R. Soc. A, Vol.270, No.27, pp. 417-434

Courjon, D. (2003). Near-Field Microscopy and Near-Field Optics, Imperial College Press, London, UK 
Dinelli, F.; Assender, H. E.; Takeda, N.; Briggs, G. A.D. \& Kolosov, O. (1999). Elastic Mapping of Heterogeneous Nanostructures with Ultrasonic Force Microscopy. Surf. Interf. Anal. Vol. 27, No.5-6, pp. 562-567

Franke, K.; Besold, J.; Haessler, W. \& Seegebarth, C. (1994). Modification and Detection of Domains on Ferroelectric PZT Films by Scanning Force Microscopy. Surf. Sci. Lett.,Vol.302, No.1-2, pp. L283-L288

Gautier, B.; Fares, B.; Prudon, G. \& Dupuy, J. (2004). Imaging by Atomic Force Microscopy of the Electrical Properties Difference of the Facets of Oxygen-Ion-Induced Ripple Topography in Silicon. Appl. Surf. Sci., Vol.231-232, No.1, pp. 136-140

Goodman, F. O. \& Garcia, N. (1991). Roles of the Attractive and Repulsive Forces in Atomic Force Microscopy. Physical Review B,Vol.43, No.6, pp. 4728-4731

Gruverman, A.; Auciello, O.; Hatano, J. \& Tokumoto, H. (1996). Scanning Force Microscopy as a Tool for Nanoscale Study of Ferroelectric Domains. Ferroelectrics, Vol.184, No.1, pp. $11-20$

Gu, Y. Q.; Ruan, X. L.; Han, L.; Zhu, D. Z.\& Sun, X. Y. (2002). Imaging of Thermal Conductivity With Sub-Micrometer Resolution Using Scanning Thermal Microscopy. International Journal of Thermophysics, Vol.23, No.4, pp. 1115-1124

Hartmann, U. (1999). Magnetic Force Microscopy. Annu. Rev. Mater. Sci., Vol. 29, pp. 53-87

Herzer, G. (1993). Nanocrystalline Soft Magnetic Materials. Physica Scripta Vol.T49A, pp. 307-314

Hirsekorn, S.; Rabe, U.; Boub, A.\& Arnold, W. (1999). On The Contrast in Eddy Current Microscopy Using Atomic Force Microscopes. Surface and Interface Analysis, Vol.27, No.5-6, pp. 474-481

Hoffmann, B.; Houbertz, R.\&Hartmann, U. (1998). Eddy Current Microscopy. Appl. Phys. A,Vol.66, Supplement No.1, pp. S409-S413

Houck, J. R. \& Bowers, R. (1964). New Type of Flux Meter for the Measurement of High Magnetic Fields at Low Temperatures. Rev. Sci. Instrum.,Vol.35, No.9, pp. 11701172

Huang, P.; Zhang, G.; Wu, Z.; Cai, J. \& Zhou, Z. (2006). Inspection of Defects in Conductive Multi-Layered Structures by an Eddy Current Scanning Technique: Simulation and Experiments. NDTEE International,Vol.39, No.7, pp. 578-584

Hutter, J. L. \& Bechhoefer, J. (1993). Calibration of Atomic-Force Microscope Tips. Rev. Sci. Instrum., Vol.64, No.7, pp.1868-1873

Hutter, J. L. \& Bechhoefer, J. (1993). Manipulation of Van Der Waals Forces to Improve Image Resolution in Atomic Force Microscopy. J. Appl. Phys., Vol.73, No.9, pp. 41234129

Karpen, W.; Becker, R. \& Arnold, W. (1998). Characterization of Electric and Magnetic Material Properties with Eddy Current Measurements. Nondestructive Testing Evaluation,Vol.15, No.2, pp. 93-107

Kelly, K. L.; Corondo, E.; Zhao, L. L.\& Schatz, G. C. (2003). The Optical Properties of Nanoparticles: The Influence of Size, Shape, and Dielectric Environment. J. Phys. Chem. B Vol.107, No.3, pp. 668-677

Kirby, M. W.\& Lareau, J. P. (1991). Eddy Current Imaging of Aircraft Using Real Time Image Signal Processing, Proceedings of the International Conference on Nondestructive Testing and Evaluation of Composite Structures, Riga, Latvia, October 22-24, 1991 
Krakowski, M. (1982). Eddy Current Losses in Thin Circular and Rectangular Plates. Archiv für Elektrotechnik,Vol.64, No.6, pp. 307-311

Landau, L. D. \& Lifshitz, E. M. (1960). Electrodynamics of Continuous Media,Pergamon,Oxford, UK

Libby, H.L. (1971). Introduction to Electrodynamic Nondestructive Test Methods,WileyInterscience,New York, USA

Matey, J. R. \& Blanc, J. (1985). Scanning Capacitance Microscopy. J. Appl. Phys.,Vol.57, No.5, pp. $1437-1444$

Maxfield, B. W. (1969). Helicon Waves in Solids. Am. J. Phys.,Vol.37, No.3, pp. 241-269.

McHenry, M. E.jJohnson, F.; Okumura, H.; Ohkubo, T. et al. (2003). The Kinetics of Nanocrystallization and Microstructural Observations in FINEMET, NANOPERM and HITPERM Nanocomposite Magnetic Materials. Scripta Materialia, Vol.48, No.7, pp. $881-887$

Meaden, G. T. (1965). Electrical Resistance of Metals, Plenum, New York, USA

Michaels, A. M.; Nirmal, M.\& Brus, L .E. (1999). Surface Enhanced Raman Spectroscopy of Individual Rhodamine 6G Molecules on Large Ag Nanocrystals. J. Am. Chem. Soc., Vol.121, No.43, pp. 9932-9939

Murphy, W. L. \& Spalding, G. C. (1999). Range of Interactions: An Experiment in Atomic and Magnetic Force Microscopy. Am. J. Phys., Vol.67, No.10, pp. 905-908

Murray, P. T.\& Shin, E. (2008). Formation of Silver Nanoparticles by Through Thin Film Ablation. Materials Letters, Vol.62, No.28, pp. 4336-4338

Nalladega, V.; Sathish, S.; Klosterman, D.; Jata, K. V. \& Blodgett, M. P. (2007). Atomic Force Microscopy Based Eddy Current Imaging and Characterization of Composite and Nanocomposite Materials, Proceedings of SAMPE Technical Conference, Baltimore, Maryland, USA, June 3-7, 2007

Nalladega, V.; Sathish, S. \& Brar, A. S. (2008a). Characterization of Defects in Flexible Circuits with Ultrasonic Atomic Force Microscopy. Microelectronics Reliability, Vol.48, No.10, pp. 1683-1688

Nalladega, V.; Sathish, S.; Jata, K. V. \&Blodgett, M.P. (2008b). Development of Eddy Current Microscopy for High Resolution Electrical Conductivity Imaging Using Atomic Force Microscopy. Rev. Sci. Instrum., Vol.79, No.7, pp. 073705-073705-11

Nalladega, V.; Sathish, S.; Gigliotti, M. F. X.; Subramanian, P. R. \& Iorio, L. (2009). Characterization of Magnetoelastic Properties at Nanoscale Using Atomic Force Microscopy. Proceedings of $14^{\text {th }}$ International Workshop on Electromagnetic Nondestructive Evaluation, ISBN 978-1-60750-553-2, Dayton, Ohio, USA, July, 2009

Nalladega, V.; Sathish, S.; Murray, T.; Shin, E.; Jata, K. V. \& Blodgett, M. P. (2011). Experimental Investigation of Interaction of Very Low Frequency Electromagnetic Waves with Metallic Nanostructure. J. Appl. Phys., Vol.109, No.11, (June 2011), pp. 114907-114907-9

Nonnenmacher, M.; O'Boyle, M. P. \& Wickramasinghe, H. K. (1991). Kelvin Probe Force Microscopy. Appl. Phys. Lett.,Vol.58, No.25, pp. 2921-2923

Nyffenegger, R. M.; Penner, R. M. \& Schierle, R. (1997). Electrostatic Force Microscopy of Silver Nanocrystals with Nanometer-Scale Resolution. Appl. Phys. Lett.,Vol.71, No.13, pp. 1878-1880 
Oh, J. \& Nemanich, R. J. (2002). Current-voltage And Imaging of $\mathrm{TiSi}_{2}$ Islands on Si(001) Surfaces Using Conductive-Tip Atomic Force Microscopy. J. Appl. Phys.,Vol.92, No.6, pp. 3326-3331

Okamoto, H. \& Imura, K. (2006). Near-field Imaging of Optical Field Wavefunctions in Metal Nanoparticles. J. Mater. Chem., Vol.16, No.40, pp. 3920-3928

Olbrich, A.; Ebersberger, B. \& Boit, C. (1998). Conducting Atomic Force Microscopy for Nanoscale Electrical Characterization of Thin $\mathrm{SiO}_{2}$. Appl. Phys. Lett.,Vol.73, No.21, pp. 3114-3116

Petrashov, V. T. (1984). An Experimental Study of Helicon Resonance in Metals. Rep. Prog. Phys.,Vol.47, No.1, pp. 47-110

Pendry, J. B.; Holden, A. J.; Stewart, W. J.\& Youngs, I. (1996). Extremely Low Frequency Plasmons in Metallic Mesostructures. Phys. Rev. Lett., Vol.76, No.25, pp. 4773-4776

Poltz, J. (1983). On Eddy Currents in Thin Plates. Archiv für Elektrotechnik, Vol.66, No.4, pp. 225-229

Polushkin, N. I.; Rao, K. V.; Wittborn, J.; Alexeev, A. M. \& Popkov, A. F. (2003). Visualization of Small Magnetic Entities by Nonmagnetic Probes of Atomic Force Microscope. J. Magn. Magn. Mater. Vol.258-259, No.1, pp. 29-31

Rabe, U. \& Arnold, W. (1994). Acoustic Microscopy by Atomic Force Microscopy. Appl. Phys. Lett., Vol. 64, No.12, pp. 1493-1495

Ruskell, T. G.; Workman, R. K.; Chen, D.; Sarid, D.; Dahl, S. \& Gilbert, S. (1996). High Resolution Fowler-Nordheim Field Emission Maps of Thin Silicon Oxide Layers. Appl. Phys. Lett.,Vol.68, No.1, pp. 93-95

Sader, J. E. (1995). Parallel Beam Approximation for V-Shaped Atomic Force Cantilevers. Rev. Sci. Instrum.,Vol.66, No.9, pp. 4583-4587

Siddoju, A.; Sathish, S.; Ko, R. \& Blodgett, M. P. (2006). Electrical Circuit Model of an Eddy Current System for Computing Multiple Parameters. AIP Conference Proceedings Review of Progress in Quantitative Nondestructive Evaluation, ISBN 978-0-7354-0399-4, Portland, Oregon, USA, July 30-August 4, 2006

Ślawska-Waniewska, A. \& Lachowicz, H. K. (2003). Magnetostriction in Soft Magnetic Nanocrystalline Materials. Scripta Materialia,Vol.48, No.7, pp. 889-894

Stern, J. E.; Terris, B. D.; Mamin, H. J.\& Rugar, D. (1988). Deposition and Imaging of Localized Charge on Insulator Surfaces Using a Force Microscope. Appl. Phys. Lett.,Vol.53, No.26, pp. 2717-2719

Takata, K. \& Tomiyama, F. (2000). Strain Imaging of a Magnetic Material. Jpn. J. Appl. Phys., Vol.39, No.5B, pp. 3090-3092

Taylor, M. T.; Merrill, J. R.\& Bowers, R. (1963). Low-frequency Magnetoplasma Resonance in Metals. Phys. Rev.,Vol.129, No.6, pp. 2525-2529

Tominaga, J.; Mihalcea, C.; Büchel, D.; Fukuda, H. et al. (2001). Local Plasmon Photonic Transistor. Appl. Phys. Lett.,Vol.78, No.17, pp. 2417-2419

Wadas, A. \& Hug, H. J. (1992). Models For The Stray Field From Magnetic Tips Used in Magnetic Force Microscopy. J. Appl. Phys.,Vol.72, No.1, pp. 203-206

Weaver, J. M. R. \& Abraham, D. W. (1991). High Resolution Atomic Force Microscopy Potentiometry. J. Vac. Sci. Technol. B, Vol.9, No.3, pp. 1559-1561

Wittborn, J.; Rao, K. V.; Nogues, J. \& Schuller. I. K. (2000). Magnetic Domain and Domainwall Imaging of Submicron Co Dots by Probing the Magnetostrictive Response Using Atomic Force Microscopy. Appl. Phys. Lett., Vol.76, No.20, pp. 2931-2933 
Williams, C.C. (1999). Two-dimensional Dopant Profiling by Scanning Capacitance Microscopy. Annu. Rev. Mater. Sci., Vol.29, pp. 471-504

Yamanaka, K.; Ogiso, H. \& Kolosov, O. (1994).Ultrasonic Force Microscopy for Nanometer Resolution Subsurface Imaging. Appl. Phys. Lett., Vol. 64, No.2, pp. 178-180.

Yoshizawa, Y.; Oguma, S. \& Yamauchi, K. (1988). New Fe-based Soft Magnetic Alloys Composed of Ultrafine Grain Structure. J. Appl. Phys.,Vol.64, No.10, pp. 6044-6046 


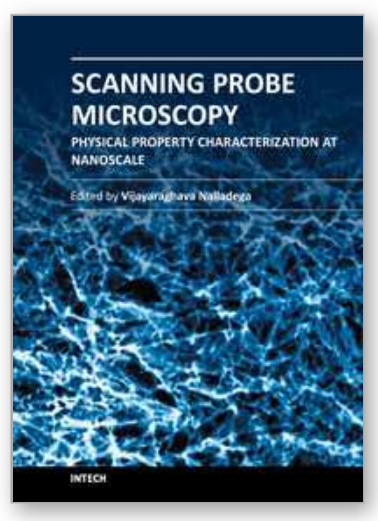

\section{Scanning Probe Microscopy-Physical Property Characterization at Nanoscale}

Edited by Dr. Vijay Nalladega

ISBN 978-953-51-0576-3

Hard cover, 242 pages

Publisher InTech

Published online 27, April, 2012

Published in print edition April, 2012

Scanning probe microscopy (SPM) is one of the key enabling tools for the advancement for nanotechnology with applications in many interdisciplinary research areas. This book presents selected original research works on the application of scanning probe microscopy techniques for the characterization of physical properties of different materials at the nanoscale. The topics in the book range from surface morphology analysis of thin film structures, oxide thin layers and superconducting structures, novel scanning probe microscopy techniques for characterization of mechanical and electrical properties, evaluation of mechanical and tribological properties of hybrid coatings and thin films. The variety of topics chosen for the book underlines the strong interdisciplinary nature of the research work in the field of scanning probe microscopy.

\section{How to reference}

In order to correctly reference this scholarly work, feel free to copy and paste the following:

Vijay Nalladega, Shamachary Sathish, Kumar V. Jata and Mark P. Blodgett (2012). Multiple Material Property Characterization Using Induced Currents and Atomic Force Microscopy, Scanning Probe Microscopy-Physical Property Characterization at Nanoscale, Dr. Vijay Nalladega (Ed.), ISBN: 978-953-51-0576-3, InTech, Available from: http://www.intechopen.com/books/scanning-probe-microscopy-physical-propertycharacterization-at-nanoscale/multiple-material-property-characterization-using-induced-currents-and-atomicforce-microscopy

\section{INTECH}

open science | open minds

\section{InTech Europe}

University Campus STeP Ri

Slavka Krautzeka 83/A

51000 Rijeka, Croatia

Phone: +385 (51) 770447

Fax: +385 (51) 686166

www.intechopen.com

\section{InTech China}

Unit 405, Office Block, Hotel Equatorial Shanghai

No.65, Yan An Road (West), Shanghai, 200040, China

中国上海市延安西路65号上海国际贵都大饭店办公楼 405 单元

Phone: +86-21-62489820

Fax: $+86-21-62489821$ 
(C) 2012 The Author(s). Licensee IntechOpen. This is an open access article distributed under the terms of the Creative Commons Attribution 3.0 License, which permits unrestricted use, distribution, and reproduction in any medium, provided the original work is properly cited. 